\title{
High-throughput screening for natural compound- based autophagy modulators reveals novel chemotherapeutic mode of action for arzanol
}

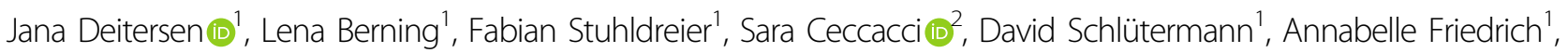

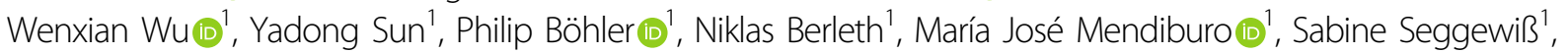

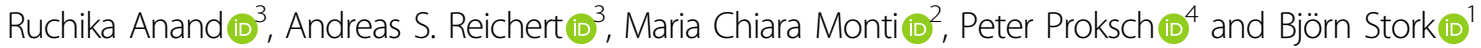

\begin{abstract}
Autophagy is an intracellular recycling pathway with implications for intracellular homeostasis and cell survival. Its pharmacological modulation can aid chemotherapy by sensitizing cancer cells toward approved drugs and overcoming chemoresistance. Recent translational data on autophagy modulators show promising results in reducing tumor growth and metastasis, but also reveal a need for more specific compounds and novel lead structures. Here, we searched for such autophagy-modulating compounds in a flow cytometry-based high-throughput screening of an inhouse natural compound library. We successfully identified novel inducers and inhibitors of the autophagic pathway. Among these, we identified arzanol as an autophagy-modulating drug that causes the accumulation of ATG16L1positive structures, while it also induces the accumulation of lipidated LC3. Surprisingly, we observed a reduction of the size of autophagosomes compared to the bafilomycin control and a pronounced accumulation of p62/SQSTM1 in response to arzanol treatment in HeLa cells. We, therefore, speculate that arzanol acts both as an inducer of early autophagosome biogenesis and as an inhibitor of later autophagy events. We further show that arzanol is able to sensitize RT-112 bladder cancer cells towards cisplatin (CDDP). Its anticancer activity was confirmed in monotherapy against both CDDP-sensitive and -resistant bladder cancer cells. We classified arzanol as a novel mitotoxin that induces the fragmentation of mitochondria, and we identified a series of targets for arzanol that involve proteins of the class of mitochondria-associated quinone-binding oxidoreductases. Collectively, our results suggest arzanol as a valuable tool for autophagy research and as a lead compound for drug development in cancer therapy.
\end{abstract}

\section{Introduction}

The discovery of approved anticancer drugs falls behind the rapid increase in cancer-related mortality. Cancer drug discovery, therefore, focusses on repurposing approved and identifying novel bioactive compounds that can serve as lead compounds. Most drugs developed over

\footnotetext{
Correspondence: Björn Stork (bjoern.stork@hhu.de)

${ }^{1}$ Institute of Molecular Medicine I, Medical Faculty and University Hospital Düsseldorf, Heinrich-Heine-University Düsseldorf, Universitätsstraße 1, 40225 Düsseldorf, Germany

${ }^{2}$ Department of Pharmacy, University of Salerno, Via Giovanni Paolo II 132 . 84084 Salerno, Fisciano, Italy

Full list of author information is available at the end of the article

Edited by G.M. Fimia
}

the last decades are based on natural compounds ${ }^{1,2}$. Their advantages lie in the inexhaustible quantity of compounds available in nature, their often non-synthesizable complexity, and a high degree of stereochemistry ${ }^{3}$. They often target evolutionarily conserved pathways that regulate cell fate decisions such as apoptosis, necrosis, senescence, or autophagy.

Autophagy is a lysosomal pathway with a mainly cytoprotective purpose that plays a role in different human pathologies such as neurodegeneration or cancer ${ }^{4-7}$. The main characteristic of (macro-)autophagy is the sequestration of aggregates, long-lived proteins, or damaged organelles by an autophagosomal membrane and its

\section{(c) The Author(s) 2021}

\footnotetext{
(c) Open Access This article is licensed under a Creative Commons Attribution 4.0 International License, which permits use, sharing, adaptation, distribution and reproduction cc) in any medium or format, as long as you give appropriate credit to the original author(s) and the source, provide a link to the Creative Commons license, and indicate if changes were made. The images or other third party material in this article are included in the article's Creative Commons license, unless indicated otherwise in a credit line to the material. If material is not included in the article's Creative Commons license and your intended use is not permitted by statutory regulation or exceeds the permitted use, you will need to obtain permission directly from the copyright holder. To view a copy of this license, visit http://creativecommons.org/licenses/by/4.0/.
} 
subsequent fusion with the lysosome for the degradation of bulk or selected cargo. Autophagy is basally active in most cell types, but can also be induced by growth factor or nutrient deficiency, hypoxia, aggregates, or mitochondrial damage ${ }^{8-10}$. Dependent on the stimulus, specific autophagy receptors help to eliminate sources of damage such as bacterial or viral components in xenophagy or damaged mitochondria in mitophagy.

In canonical autophagy, 5'-AMP-activated protein kinase (AMPK)-dependent activation and mammalian target of rapamycin (mTOR)-dependent inhibition regulate the autophagy-initiating unc-51-like kinase 1 (ULK1) complex. Upon induction of autophagy, the ULK1 complex activates the class III phosphatidylinositol 3kinase (PIK3C3/VPS34) complex that produces phosphatidylinositol 3-phosphate (PI3P) at subregions of the $\mathrm{ER}^{11}$. ATG9A as well as the PI3P-binding proteins DCFP1 and WIPI1/2 are involved in membrane elongation and recruit the ubiquitin-like ATG12-ATG5-ATG16L1 complex. Together with ATG7 and ATG3, the ATG12ATG5-ATG16L1 complex conjugates phosphatidylethanolamine to LC3 (ref. ${ }^{12}$ ). Lipidated LC3 decorates autophagosomal membranes and serves as an "anchor" for the recruitment of autophagosomal cargo or downstream autophagy regulators. For instance, LC3 binds p62 (also known as sequestosome 1, SQSTM1), which is an autophagic receptor for ubiquitinated $\operatorname{cargo}^{13}$. After fusion of autophagosome and lysosome, lysosomal hydrolases degrade not only autophagic cargo but also LC3 and LC3-bound receptors such as p62/SQSTM1. Accordingly, a reduction in both LC3 and p62/SQSTM1 levels serves as a readout for autophagic flux.

Autophagy ensures cell survival and prevents the accumulation of carcinogenic stimuli in cells, suggesting a protective role of autophagy against tumorigenesis. In late-stage cancer, on the other hand, autophagy can be tumor-promoting due to its catabolic function, supporting solid tumors in hypoxic regions and enabling the tumor microenvironment to contribute nutrients and growth factors ${ }^{14-18}$. In these tumors, autophagy has been found to contribute to cancer cell survival and poor outcome ${ }^{19,20}$. Recent attempts in translational cancer research, therefore, investigate the inhibition of autophagy in both monotherapy and combinational treatment in order to sensitize cancer cells to chemotherapy ${ }^{21-24}$. In contrast, inducers of autophagy are also discussed as potential chemotherapeutics, driving cancer cells into autophagy-associated or autophagic cell death ${ }^{25-29}$.

This work investigates an in-house library of natural compounds in order to find modulators of autophagy, which can be applied as novel chemotherapeutics for monotherapy or combinational therapy. We found that arzanol, a phloroglucinol derivative isolated from the plant Helichrysum italicum (Asteraceae), impairs the viability of bladder cancer cells, and we investigated its molecular mode of action. We identify arzanol as dual modulator of autophagy and expand the list of arzanol targets by mitochondria-related oxidoreductases and autophagy-related proteins. Taken together, we propose arzanol as a new tool to study autophagy and as a potential lead structure for combinational chemotherapy with CDDP in urothelial bladder carcinoma cells.

\section{Results \\ High-throughput screening reveals modulators of autophagy}

To identify novel modulators of autophagy among natural compounds, we screened an in-house library of 300 natural compounds derived from marine sponges, endophytic fungi, and higher plants, using a flow cytometric high-throughput screening (Fig. 1). The autophagic flux was measured in mouse embryonic fibroblasts (MEFs) expressing mCitrine-tagged LC3. As growth medium, we used serum-supplied full medium including amino acids, while for starvation, we used serum-free and amino acid-deficient starvation medium. During serumand amino acid starvation, mCitrine-LC3 anchored to the inner autophagosomal membrane is degraded by lysosomal hydrolases, causing a reduction of the mCitrine-LC3 signal (Fig. 1A). Upon treatment with the lysosomal inhibitor bafilomycin $A_{1}$, both basal and starvationinduced autophagic flux is inhibited and the mCitrineLC3 signal increases (Fig. 1A), confirming the applicability of this assay to identify both inducers and inhibitors of autophagy.

For the classification of inducers and inhibitors, we set the threshold at a $15 \%$ decrease or increase of the mCitrine-LC3 signal upon treatment with the compounds. This threshold value is based on the positive control for autophagy induction in our cellular model system, i.e., Torin 2 treatment for $6 \mathrm{~h}$. In addition, we set our threshold to compounds with a fairly moderate and higher effect according to statistical analyses of highthroughput data ( $\mid$ SSMD score $\mid \geq 1$ ) (Fig. 1, Tables 1 and 2 , and Supplementary Table 1$)^{30}$. In doing so, the screening of MEFs treated with natural compounds in full medium revealed five potential inhibitors of basal autophagy (Fig. 1B) and eight potential inducers of autophagy (Fig. 1B and Table 1). The screening in starvation medium revealed 64 potential inhibitors of autophagic flux (Fig. 1C and Table 2). In addition to the aforementioned five inhibitors, and four dual modulators (i.e., activating in full medium, but inhibitory under starvation), 55 compounds were classified as potential autophagy inhibitors. Of the 300 tested natural compounds, two-thirds did not have a considerable effect on autophagy (Fig. 1D). 
A
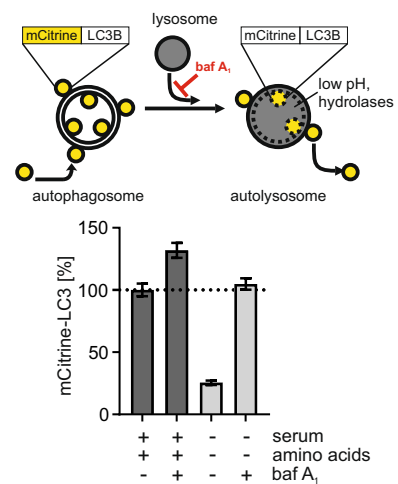

B

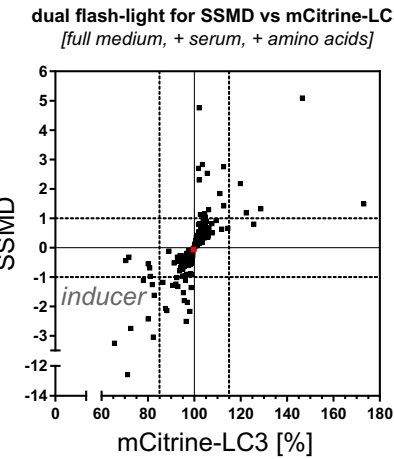

C

dual flash-light for SSMD vs mCitrine-LC3 [starvation medium, - serum, - amino acids]

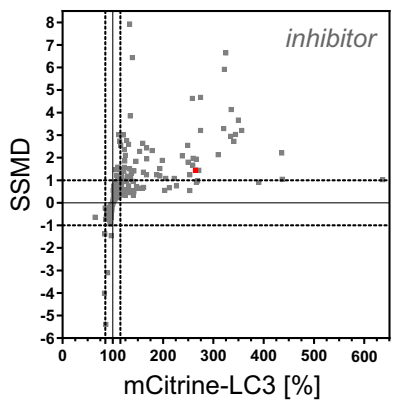

D $\quad 200$ full medium
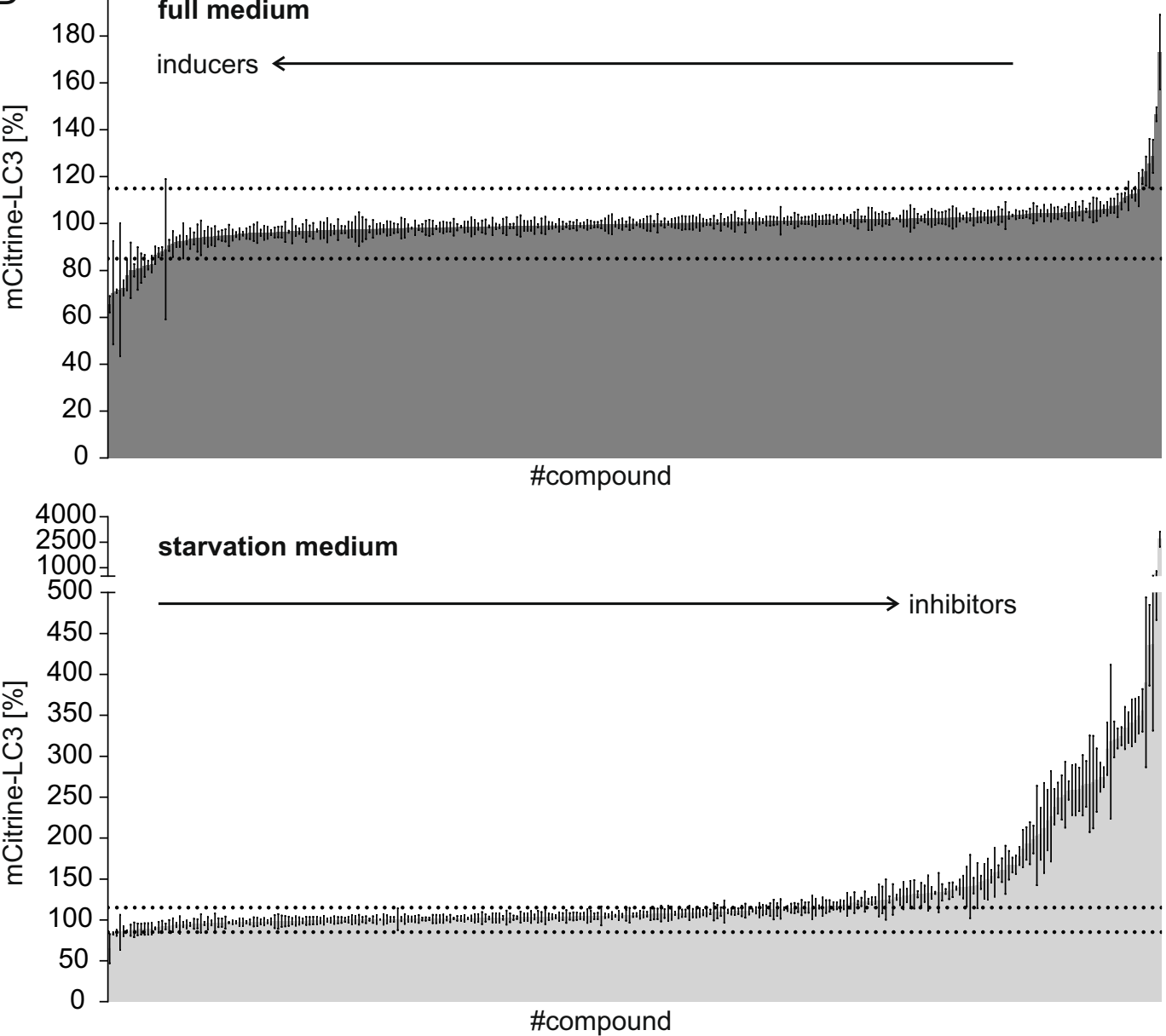

Fig. 1 High-throughput screening for natural compound-based autophagy modulators. A Detection of starvation-induced and bafilomycin $A_{1}$-inhibited autophagy in a flow cytometric screening. MEFs stably expressing mCitrine-LC3 were cultured either in full medium plus serum or in starvation medium and were treated with either $10 \mathrm{nM}$ bafilomycin $\mathrm{A}_{1}$ or mock treatment (DMSO) for $6 \mathrm{~h}(n \geq 19)$. The mCitrine fluorescence is shown as a percentage relative to that of mock-treated cells. Data represent means \pm SEM. B, C High-throughput autophagy screening of 300 natural compounds. The diagram shows dual-flashlight plots for strictly standardized mean difference (SSMD) versus the average percentage of mCitrineLC3. Dotted lines define potential inducers and inhibitors of autophagy. Cells were treated with $10 \mu \mathrm{M}$ of each compound for $6 \mathrm{~h}(n=3)$. Arzanol is highlighted in red. D The diagram shows individual levels of mCitrine-LC3 fluorescence from (B, C) for each of the 300 compounds in full or starvation medium. Dotted lines mark the $\pm 15 \%$ difference in mCitrine-LC3 levels. Data represent means \pm SEM. 
Table 1 List of potential autophagy inducers.

\begin{tabular}{llrr}
\hline Compound & Mean difference of mCit-LC3 (\%) & SD & SSMD \\
\hline P05E07 & -34.4 & \pm 6.0 & -3.25 \\
P02E06 & -28.8 & \pm 1.3 & -12.58 \\
P04C02 & -27.4 & \pm 5.6 & -2.75 \\
P01F03 & -22.0 & \pm 11.2 & -1.11 \\
P05C06 & -19.9 & \pm 4.6 & -2.43 \\
P01G08 & -18.1 & \pm 8.1 & -1.26 \\
P03F08 & -17.7 & \pm 3.3 & -3.04 \\
P02B03 & -17.3 & \pm 6.0 & -1.62 \\
\hline
\end{tabular}

SD standard deviation SSMD strictly standardized mean difference.

In total, eight compounds causing equal to or greater than 15\% LC3 degradation at a fairly moderate and higher effect ( $\mid$ SSMD score $\mid \geq 1$ ) at $10 \mu \mathrm{M}$ after $6 \mathrm{~h}$ of treatment were classified as potential inducers of autophagy.

Data show mean difference \pm SD (\% of control) of mCitrine-LC3 decrease upon full medium monitored by flow cytometry.

\section{Arzanol interferes with late-stage autophagy}

Among the novel modulators of autophagy, we identified the phloroglucinol $\alpha$-pyrone arzanol (Fig. 2A; marked as red dots in Fig. $1 \mathrm{~B}, \mathrm{C}$ ), which was isolated from the aerial parts of Helichrysum italicum ${ }^{31}$. We deliberately chose arzanol for further analysis because of the following reasons: (1) lack of significant cytotoxicity in the cell line used for screening, (2) identification of autophagymodulating properties in orthogonal assays (e.g., LC3 turnover by immunoblotting, see below), (3) sufficient stability, (4) bioaccessibility, (5) price/commercial availability, and (6) lack of previous publications. Arzanol is known for its anti-inflammatory, antiviral ${ }^{32}$, antioxidative, and cytotoxic effects ${ }^{33}$, however, no effect on autophagy has been reported before. To identify the pathway affected by arzanol, we performed immunoblotting analyses of key components of the canonical autophagy pathway such as components of the mTOR-ULK1-axis (i.e., phosphorylation of ribosomal protein S6 kinase beta-1 (p70S6K) at Ser371 and ULK1 at Ser758), LC3, and p62/SQSTM1. During the early biogenesis of autophagosomes, cytosolic LC3-I gets lipidated to autophagosomal membranebound LC3-II, which is-along with the autophagy receptor p62/SQSTM1-later degraded by lysosomal hydrolases ${ }^{34}$.

We observed that arzanol does not inhibit starvationinduced inactivation of mTOR; however, it causes the accumulation of LC3-II and p62 during starvationinduced autophagy (Fig. 2B-F). We validated these effects using commercially available arzanol (Fig. $2 \mathrm{H}$ ). The effect of arzanol on p62/SQSTM1 accumulation was more prominent upon amino acid starvation (Fig. 2I). We further validated the accumulation of LC3 in HeLa cells expressing the GFP-LC3-RFP-LC3 $\Delta G$ reporter construct $^{35}$ by flow cytometry, measuring the autophagic
Table 2 List of potential autophagy inhibitors.

\begin{tabular}{|c|c|c|c|}
\hline Compound & Mean difference of mCit-LC3 (\%) & SD & SSMD \\
\hline P05D05 & $+2590.4^{\mathrm{a}}$ & \pm 765.1 & 1.91 \\
\hline P05C04 & +536.3 & \pm 294.2 & 1.03 \\
\hline P02D03 & +336.7 & \pm 182.2 & 1.04 \\
\hline P05D10 & +335.7 & \pm 85.5 & 2.21 \\
\hline $\mathrm{P} 04 \mathrm{C} 10$ & +256.2 & \pm 45.0 & 3.21 \\
\hline P01B11 & +249.9 & \pm 38.4 & 3.67 \\
\hline Р03В08 & +243.9 & \pm 45.4 & 3.03 \\
\hline P01F02 & +240.8 & \pm 49.9 & 2.72 \\
\hline Р03В06 & +235.2 & \pm 32.0 & 4.14 \\
\hline P05B05 & $+234.4^{\mathrm{a}}$ & \pm 44.8 & 2.95 \\
\hline P01E05 & +224.6 & \pm 19.1 & 6.65 \\
\hline P05E10 & +221.8 & \pm 21.1 & 5.92 \\
\hline P01C06 & +220.6 & \pm 37.8 & 3.29 \\
\hline P01F11 & +209.4 & \pm 55.6 & 2.13 \\
\hline P01G06 & +174.5 & \pm 21.1 & 4.67 \\
\hline P04E03 & +174.3 & \pm 30.7 & 3.21 \\
\hline P02D05 & +170.9 & \pm 67.0 & 1.44 \\
\hline P04E04 & +166.1 & \pm 48.5 & 1.93 \\
\hline Arzanol & +164.6 & \pm 63.9 & 1.45 \\
\hline P01F08 & +159.6 & \pm 45.8 & 1.97 \\
\hline Р05B09 & +158.8 & \pm 53.8 & 1.67 \\
\hline P01G09 & +158.7 & \pm 52.9 & 1.69 \\
\hline P04D02 & +158.2 & \pm 19.3 & 4.63 \\
\hline Р05B06 & +153.1 & \pm 69.7 & 1.24 \\
\hline Р05B02 & +149.6 & \pm 47.0 & 1.80 \\
\hline P05C03 & +149.0 & \pm 32.9 & 2.55 \\
\hline P03F09 & +138.3 & \pm 37.5 & 2.08 \\
\hline P02E04 & +122.0 & \pm 63.9 & 1.08 \\
\hline P02F04 & +105.1 & \pm 55.0 & 1.08 \\
\hline P04G07 & +98.3 & \pm 29.4 & 1.88 \\
\hline Р02B07 & +93.7 & \pm 44.5 & 1.19 \\
\hline Р04B04 & +93.4 & \pm 34.7 & 1.52 \\
\hline Р04В07 & +87.2 & \pm 39.5 & 1.24 \\
\hline P04F06 & +78.1 & \pm 19.0 & 2.32 \\
\hline P05E07 & +67.4 & \pm 19.5 & 1.95 \\
\hline P04B02 & +67.1 & \pm 15.6 & 2.43 \\
\hline P04G08 & +66.9 & \pm 30.5 & 1.24 \\
\hline P02B02 & +60.7 & \pm 25.1 & 1.36 \\
\hline P05F02 & +59.4 & \pm 12.7 & 2.64 \\
\hline
\end{tabular}


Table 2 continued

\begin{tabular}{|c|c|c|c|}
\hline Compound & Mean difference of mCit-LC3 (\%) & SD & SSMD \\
\hline P03F08 & +53.3 & \pm 13.2 & 2.28 \\
\hline P05F03 & +46.0 & \pm 14.1 & 1.84 \\
\hline P01C05 & +41.8 & \pm 16.6 & 1.42 \\
\hline $\mathrm{P} 04 \mathrm{C02}$ & +40.0 & \pm 16.0 & 1.41 \\
\hline P02E08 & +38.7 & \pm 3.4 & 6.44 \\
\hline P05E06 & +36.9 & \pm 15.9 & 1.31 \\
\hline P04E10 & +35.4 & \pm 17.2 & 1.16 \\
\hline P05C06 & +34.8 & \pm 5.1 & 3.85 \\
\hline P03E07 & +33.6 & \pm 17.6 & 1.08 \\
\hline P05D06 & +33.2 & \pm 12.4 & 1.51 \\
\hline P05G05 & +33.2 & \pm 2.4 & 7.93 \\
\hline P02D07 & +32.1 & \pm 17.3 & 1.05 \\
\hline P05E04 & +31.3 & \pm 6.8 & 2.59 \\
\hline P01G02 & +29.8 & \pm 14.2 & 1.18 \\
\hline P04C11 & +27.6 & \pm 5.6 & 2.76 \\
\hline P01C09 & +25.1 & \pm 6.8 & 2.10 \\
\hline P05F04 & +23.8 & \pm 7.2 & 1.86 \\
\hline P04B10 & +23.1 & \pm 8.3 & 1.57 \\
\hline P05D07 & +22.9 & \pm 5.5 & 2.36 \\
\hline P01E03 & +22.0 & \pm 9.0 & 1.39 \\
\hline P05E02 & +21.1 & \pm 4.0 & 3.01 \\
\hline P04E02 & +18.2 & \pm 9.2 & 1.12 \\
\hline P04D04 & +17.2 & \pm 8.0 & 1.21 \\
\hline P05B10 & +17.2 & \pm 7.5 & 1.30 \\
\hline P03E06 & +15.9 & \pm 3.5 & 2.56 \\
\hline
\end{tabular}

SD standard deviation, SSMD strictly standardized mean difference. ${ }^{\text {a }}$ Could be caused by autofluorescence.

In total, 64 compounds causing equal to or greater than 15\% LC3 protection at a fairly moderate and higher effect (|SSMD score $\mid \geq 1)$ at $10 \mu \mathrm{M}$ after $6 \mathrm{~h}$ of treatment were classified as potential inhibitors of autophagy.

Data show mean difference \pm SD (\% of control) of mCitrine-LC3 increase upon starvation medium monitored by flow cytometry.

degradation of GFP-LC3 (Fig. 2J). The effect of arzanol on autophagy is consistent within all assays (i.e., murine vs. human cells, immunoblotting vs. flow cytometry, and selfisolated vs. commercial arzanol), which is why we used commercially available arzanol in all further experiments. Of note, we found that arzanol was ineffective in serumsupplied growth medium due to its serum-binding capacity (Supplementary Fig. 1A), which also explains the absence of effects in our screening in full medium (Fig. 1B). In serum-free and amino acid-rich medium, however, arzanol caused accumulation of LC3-II, which resembles its effects in the amino acid-free starvation medium (Fig. 2H). Furthermore, we exclude the involvement of antioxidative or scavenging effects of arzanol during amino acid starvation to be the reason for the observed effects, although antioxidative effects were observed in serum-free and amino acid-rich medium (Supplementary Fig. 1B).

\section{Arzanol changes ATG16L1 localization}

An increase in LC3 can be indicative of disrupted autophagic degradation or increased protein expression, while an increase in LC3-II can additionally hint to its enhanced lipidation. To determine the circumstances that led to the accumulation of LC3 and p62/SQSTM1 upon serum- and amino acid starvation (Fig. 2), we detected both overexpressed GFP-LC3 (Fig. 3A) and endogenous LC3 (Fig. 3B) using fluorescence microscopy and quantified both size and amount of LC3-positive dots that commonly represent autophagosomes ${ }^{36}$. A clear trend toward increasing numbers of LC3 dots upon arzanol treatment was observed (Fig. 3A, B). However, the size of these LC3 dots appeared rather small, making them partly indistinguishable for the quantification software. Nevertheless, we think one can appreciate the increase in the number of both overexpressed and endogenous LC3 dots and their significantly smaller size compared to bafilomycin $\mathrm{A}_{1}$-treated cells (Fig. 3A, B; i and ii.). Further, we examined the location, amount, and size of ATG16L1 dots, which is a core component of the LC3 lipidation machinery. Arzanol treatment caused a significant increase in the number of ATG16L1-positive structures (Fig. $3 \mathrm{Ci}$ ), while the size of the dots and the total protein level of ATG16L1 remained unaltered (Fig. 3Cii and data not shown). Further, some of these ATG16L1 dots show a perinuclear localization, and are surrounded by a dense population of LC3-positive dots (Supplementary Fig. 2A). During autophagosomal membrane expansion and cargo sequestration, ATG16L1 is recruited to PE-containing phagophores by PI3P-binding WIPI2 (refs. ${ }^{37,38}$ ). Of note, we did not observe an increased number of WIPI2 dots in response to arzanol (Supplementary Fig. 2B). Taken together, these data are in line with increased LC3-II in response to arzanol treatment we described above (Fig. $2 \mathrm{E}, \mathrm{H}, \mathrm{J}$ ) and suggest an altered elongation process of autophagosomes upon arzanol treatment. Finally, we also investigated autophagic flux using the combination of arzanol with bafilomycin $\mathrm{A}_{1}$ (Supplementary Fig. 2C). We did not observe any differences between the combined treatment and the treatment with bafilomycin $\mathrm{A}_{1}$ alone, indicating that arzanol ultimately blocks autophagic flux.

\section{Arzanol as an anticancer drug in mono- and CDDP- combination therapy}

In addition to monotherapy, combinational therapies of approved drugs with autophagy modulators have been proposed to overcome tumor survival ${ }^{39-41}$. In order to 


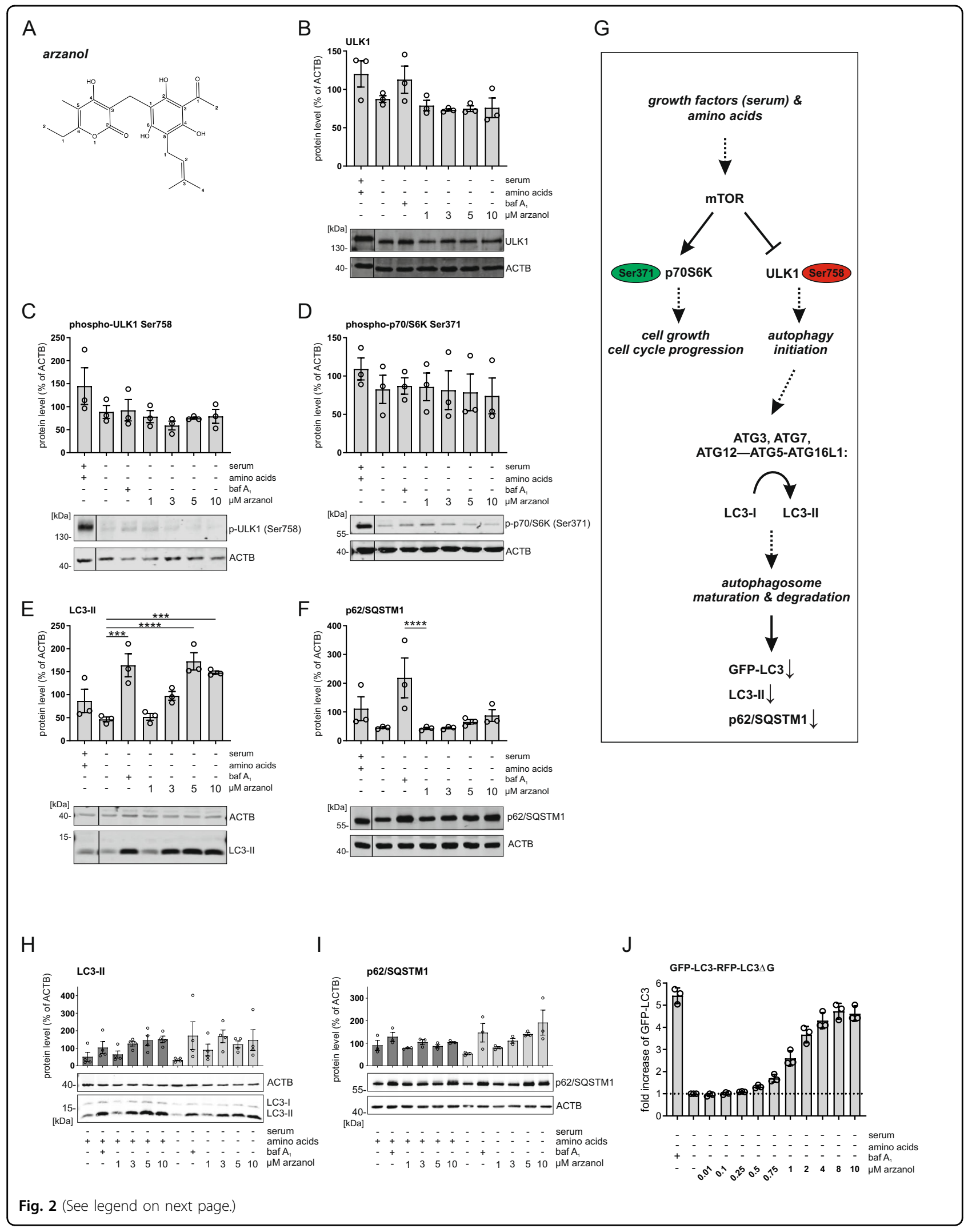


(see figure on previous page)

Fig. 2 Arzanol drives LC3-II accumulation independent of the AKT/mTOR-pathway. A Structure of arzanol. B-F Immunoblot analysis of mTOR/ ULK1-pathway proteins during serum- and amino acid starvation. Wild-type HeLa cells were starved in serum- and amino acid-free medium for $6 \mathrm{~h}$ while being incubated with baf $A_{1}$ or different concentrations of self-isolated arzanol. Data show quantified means of biological triplicates normalized to $\beta$-actin (ACTB) \pm SEM. Representative immunoblots are additionally shown. Nonadjacent lanes (i.e., full medium control lane) are indicated by vertical black lines. Statistical analysis was performed using two-way ANOVA with Dunnett's multiple comparison test comparing treated to untreated samples, ${ }^{* * *} P<0.001,{ }^{* * * *} P<0.0001$. G Simplified pathway of key proteins of the mTOR/ULK1-axis in starvation-induced autophagy. H, I Immunoblot analysis of LC3-II and p62/SQSTM1 during serum-starvation. Wild-type HeLa cells were starved in serum-free (dark bars) or serum- and amino acid-free (light bars) medium for $6 \mathrm{~h}$ while being incubated with baf $\mathrm{A}_{1}$ or different concentrations of commercially available arzanol. Data show quantified means of biological triplicates normalized to ACTB \pm SEM. $\mathbf{J}$ HeLa cells stably expressing GFP-LC3-RFP-LC3 $\triangle$ G were starved in serum- and amino acidfree medium for $6 \mathrm{~h}$ while incubated with different concentrations of commercially available arzanol. Data show fold increase of GFP-LC3

fluorescence relative to mock-treated control as means \pm SEM of three biological replicates.

test the cytotoxic effect of arzanol on cancer cells, we performed MTT assays after $24 \mathrm{~h}$ of either arzanol monotherapy or combinational therapy with cisplatin (CDDP) in RT-112 urothelial bladder carcinoma cells. We tested the cytotoxic effect of arzanol both in serum-free growth medium containing amino acids (Fig. 4A-C) and in serum- and amino acid-deficient starvation medium (Fig. 4D-F). The results of the cell viability assays reveal that arzanol is a moderate chemotherapeutic alone during amino acid supply in both CDDP-resistant cells (IC50: $9.6 \mu \mathrm{M}$; Fig. 4A) and in CDDP-sensitive RT-112 cells (IC50: $6.6 \mu \mathrm{M}$; Fig. 4B). For the amino acid-supplied CDDP-sensitive RT-112 cells, arzanol sensitizes the cells toward CDDP treatment (IC50 shift from $22.5 \mu \mathrm{M}$ to $7.1 \mu \mathrm{M}$; Fig. 4C). Similarly, arzanol is also a moderate chemotherapeutic alone during amino acid starvation in both CDDP-resistant cells (IC50: $22.6 \mu \mathrm{M}$; Fig. 4D) and in CDDP-sensitive RT-112 cells (IC50: $13.2 \mu \mathrm{M}$; Fig. 4E). Thereby, arzanol appears less toxic during amino acid starvation in relation to the non-starved samples in both cell lines. For the amino acid-starved CDDP-sensitive RT112 cells, arzanol still sensitizes the bladder cancer cells toward CDDP treatment (IC50 shift from $20.7 \mu \mathrm{M}$ to $6.3 \mu \mathrm{M}$; Fig. 4F). In both nutrient conditions, arzanol did not sensitize the CDDP-resistant cell line (Supplementary Fig. 3A).

Surprisingly, when we compared these MTT assay data with data from an Alamar blue assay (Supplementary Fig. 3B), we noticed that especially for CDDP-resistant cells the treatment with low amounts of arzanol resulted in an increased reduction of MTT, but a decreased reduction of resazurin in the Alamar blue assay (Supplementary Fig. 3C). While both assays are colorimetric assays, the MTT assay measures the metabolic activity of enzymes that are capable to reduce the tetrazolium dye MTT 3-(4,5-dimethylthiazol2-yl)-2,5-diphenyltetrazolium bromide to its insoluble formazan, whereas the Alamar blue assay depends on enzymes that reduce resazurin to resofurin. Similarly to our results, Hamid et al. observed the same phenomenon for the NAD (P)H quinone dehydrogenase 1 (NQO1) inhibitor dicoumarol $^{42}$, which shares structural similarity with arzanol.
In contrast to their observations on dicoumarol, we still witnessed that the cells die at higher concentrations of arzanol. Nevertheless, we suspect an effect of arzanol on the oxidoreductases involved in these assays.

\section{Arzanol targets mitochondria}

In our search for upstream causes of ATG16L1 accumulation upon arzanol treatment, we next investigated mitochondrial integrity. Different mitotoxins stimulate mitophagy as a means of cell survival. They induce the fragmentation of damaged mitochondria as well as the recruitment of mitophagy-associated proteins PTENinduced putative kinase protein 1 (PINK) and the E3 ubiquitin ligase Parkin ${ }^{43-45}$. In microscopy assays, arzanol induced the fragmentation of mitochondria determined with mito-DsRed localizing to the mitochondrial matrix and staining of the translocase of outer membrane $20 \mathrm{kDa}$ subunit (TOM20) (Fig. 5A and Supplementary Fig. 4A). In line with this, arzanol caused the mitochondrial stressinduced cleavage of optic atrophy protein 1 (OPA1), presumable ubiquitination of Parkin, and stabilization of PINK primarily during amino acid starvation (Fig. 5B). Interestingly, the fragmented mitochondria seem adjacent to but not colocalized with the ATG16L1 dots observed upon arzanol treatment during serum- and amino acid starvation (Supplementary Fig. 4B).

Consistent with mitochondrial damage, we identified different mitochondrial proteins as novel protein targets for arzanol using two approaches. We performed affinity purification with arzanol covalently immobilized on beads and fished for binding-partners in HeLa and MEF cellular lysates and lysates from isolated mitochondria. We also carried out drug affinity responsive target stability (DARTS) assays in mitochondrial lysates to evaluate the enhanced stability of arzanol-binding targets against protease degradation. Both approaches were followed by mass spectrometry or immunoblotting to identify and confirm arzanol targets (Fig. 6A and Supplementary Table 2). Of note, we identified mitochondrial respiratory chain complex III proteins ubiquinol-cytochrome c reductase hinge protein (UQCRH) and cytochrome c somatic 


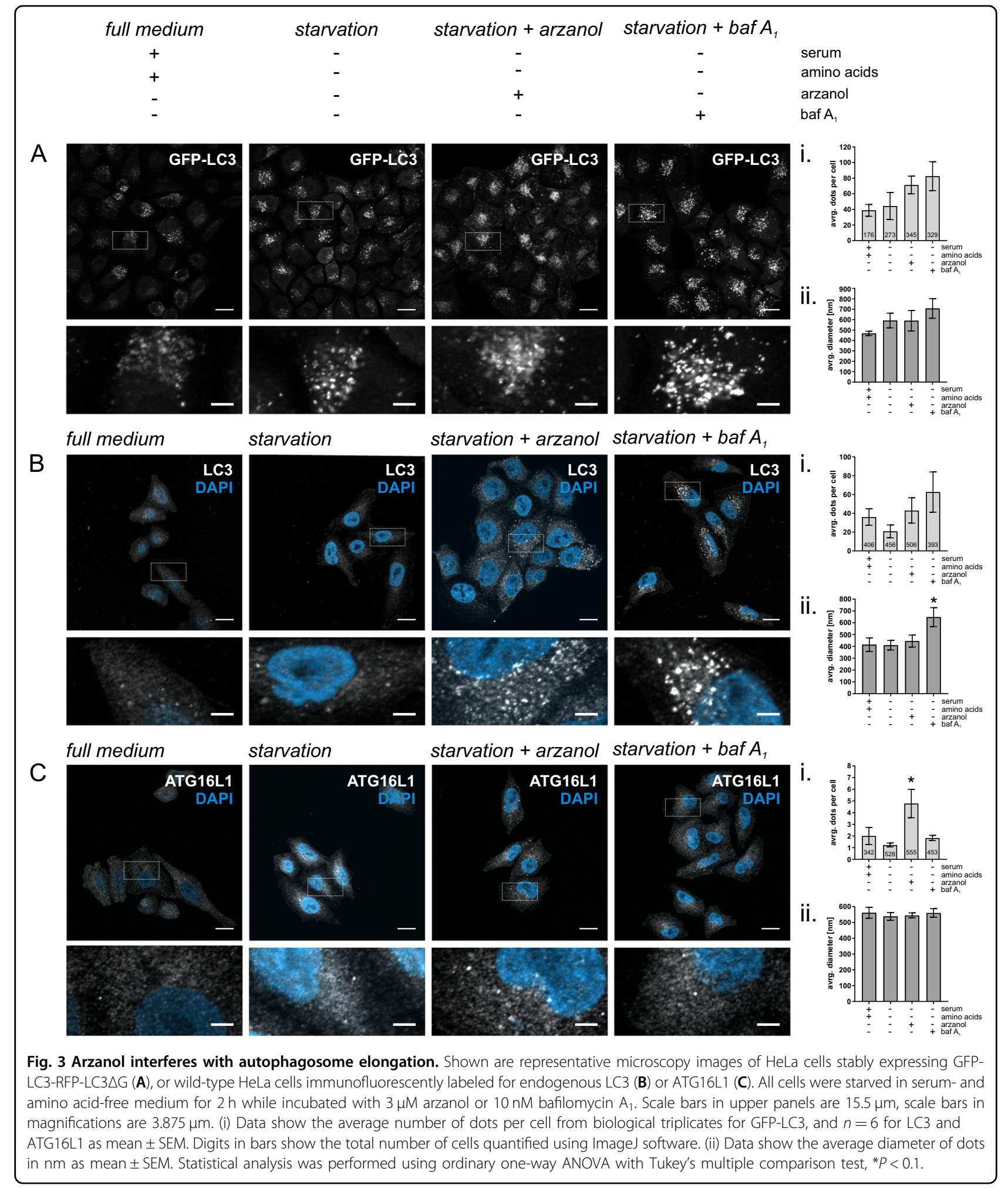

(CYCS), and complex I-related NADH:ubiquinone oxidoreductase subunit S4 (NDUFS4) bound to immobilized arzanol in affinity purification in mitochondrial lysates. We, therefore, monitored cellular oxygen consumption rates (OCR) in arzanol-treated and control cells using a Seahorse Extracellular Flux Analyzer (Fig. 6B). We observed that arzanol treatment reduced both the basal and the maximal OCR. Oligomcyin (inhibitor of $F_{1} F_{O}$ 


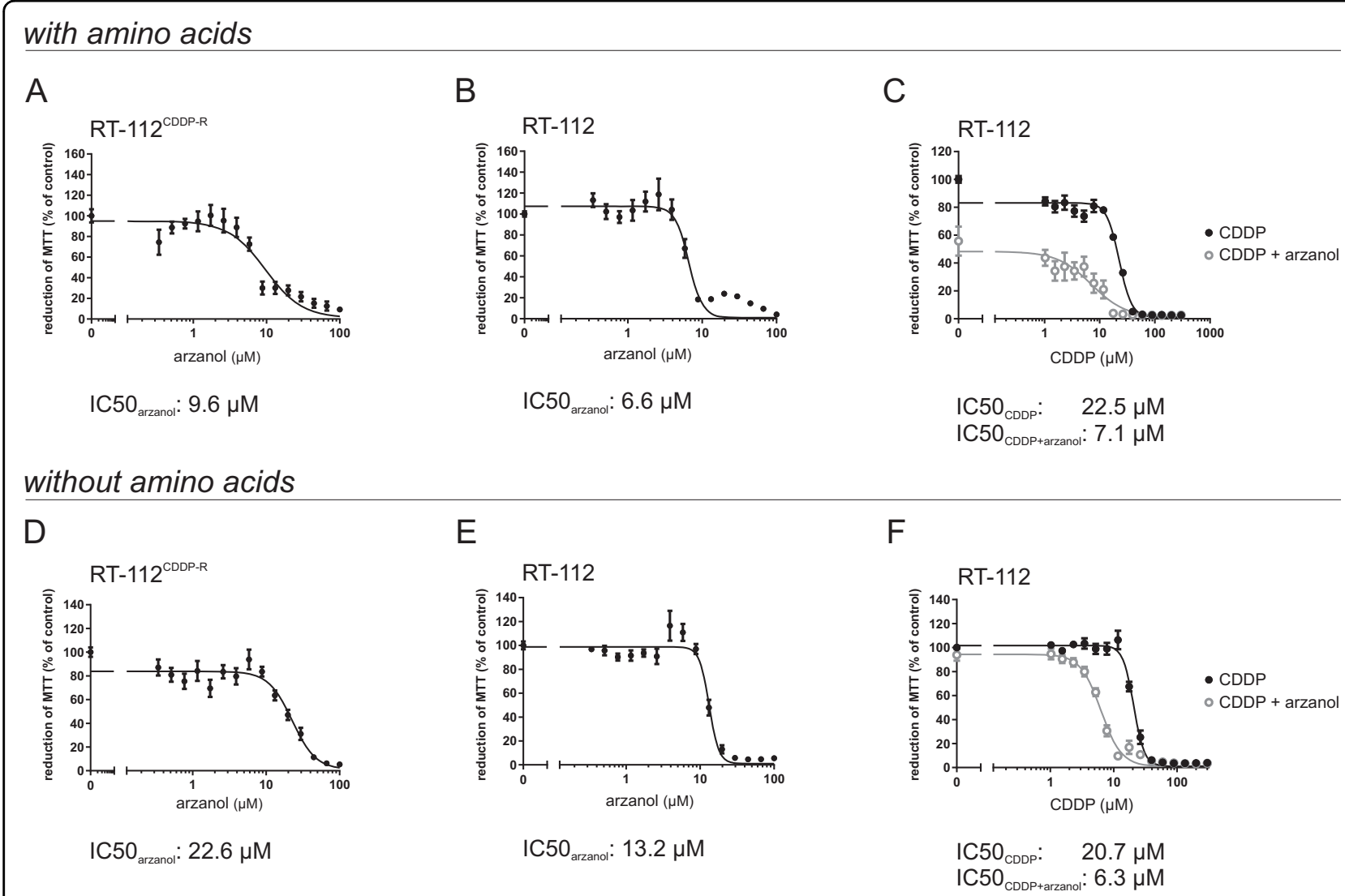

Fig. 4 Arzanol is cytotoxic for bladder carcinoma cells in mono- and CDDP-combination therapy. Arzanol reduces cell viability in bladder cancer cells as determined by the MTT assay. CDDP-resistant (CDDP-R) RT-112 bladder carcinoma cells (A) and CDDP-sensitive RT-112 bladder carcinoma cells (B) were incubated for $24 \mathrm{~h}$ with indicated concentrations of arzanol in serum-free, amino acid-supplied medium. C CDDP-sensitive RT-112 bladder carcinoma cells were incubated with indicated concentrations of CDDP in monotherapy or in a combination with $5 \mu \mathrm{M}$ arzanol in serum-free, amino acid-supplied medium. CDDP-resistant (CDDP-R) RT-112 bladder carcinoma cells (D) and CDDP-sensitive RT-112 bladder carcinoma cells $(\mathbf{E})$ were incubated for $24 \mathrm{~h}$ with indicated concentrations of arzanol in serum- and amino acid-free medium. F CDDP-sensitive RT-112 bladder carcinoma cells were incubated with indicated concentrations of CDDP in monotherapy or in a combination with $5 \mu \mathrm{M}$ arzanol in serumand amino acid-free medium. The results are shown as mean \pm SEM of at least three individual experiments performed in triplicates. $A l l ~ I C_{50}$ values were calculated using GraphPad Prism 7.01 (function log(inhibitor) vs. response - variable slope (four parameters)).

ATP synthase/complex V) did not result in a further reduction of the OCR and FCCP (uncoupler) did not stimultate cellular respiration, indicating that the oxidative phosphorylation (OXPHOS) complexes (complexes I-IV) and possibly also the $\mathrm{F}_{1} \mathrm{~F}_{\mathrm{O}}$ ATP synthase (complex V) are targeted by arzanol. We next tested arzanol directly in mitochondrial activity assays for OXPHOS complexes, and found that arzanol mostly inhibited complexes II and III, but also $\mathrm{F}_{1} \mathrm{~F}_{\mathrm{O}}$ ATP synthase/complex V (Fig. 6C). In line with these data, the succinate dehydrogenase complex iron sulfur subunit B (SDHB, OXPHOS complex II) and the ubiquinol-cytochrome $\mathrm{c}$ reductase Rieske iron-sulfur polypeptide 1 (UQCRFS1, OXPHOS complex III) were validated by immunoblotting assays of DARTS samples (Fig. 6D). Moreover, these immunoblotting assays also verified CPS1, GARS, and HSPA9 as arzanol targets. With the structural similarity of arzanol and dicoumarol, and the divergent effects of arzanol in MTT versus Alamar blue assays in mind, we also tested arzanol in a NQO1 activity assay. Arzanol reduced the activity of NQO1 in concentrations of $10 \mu \mathrm{M}$ (Fig. 6E).

Taken together, our results show that arzanol sensitizes RT-112 bladder carcinoma cells towards CDDP, and reduces cell viability of both normal and CDDP-resistant RT-112 in monotherapy. Arzanol is able to target different quinone-dependent reductases such as NQO1 and oxidoreductases of the mitochondrial respiratory chain that maintain mitochondrial integrity. Arzanol reduces mitochondrial respiration and induces fission of mitochondria independent from reactive oxygen species. We observed the accumulation of ATG16L1 and lipidated LC3, but also smaller autophagosomal structures and the accumulation of p62/SQSTM1 upon arzanol treatment during starvation. 

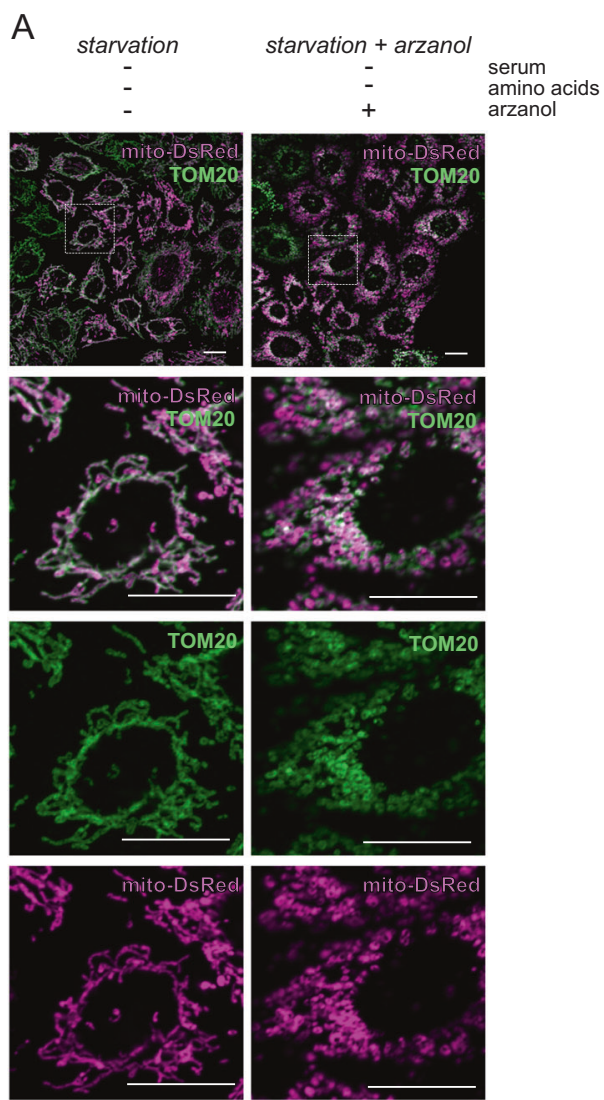

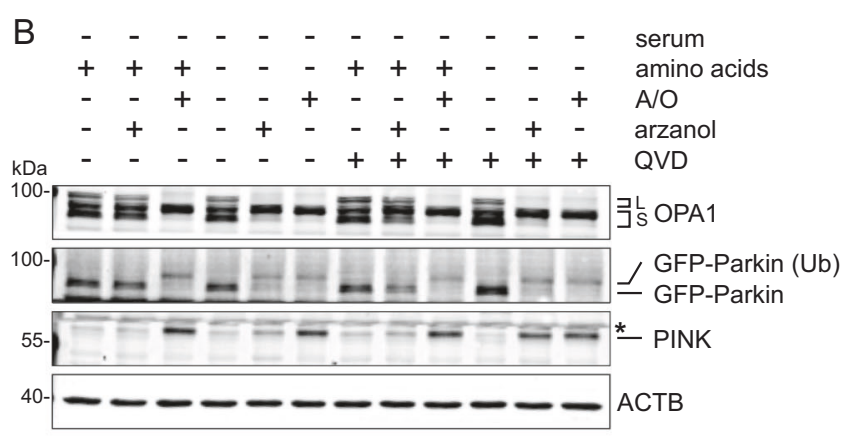

Fig. 5 Arzanol causes damage-induced fragmentation of mitochondria during starvation. A Shown are microscopy images of mito-DsRedexpressing HeLa cells immunofluorescently labeled for endogenous TOM20. During starvation, arzanol induces fragmentation of both inner (mitoDsRed) and outer (TOM20) mitochondrial membranes. Cells were starved in serum- and amino acid-free medium for $2 \mathrm{~h}$ while incubated with $5 \mu \mathrm{M}$ arzanol. Scale bars in all panels are $20 \mu \mathrm{m}$. B Immunoblot for markers of mitochondrial damage (OPA1 cleavage, Parkin ubiquitination (Ub), PINK accumulation) reveals that arzanol-induced mitochondrial fragmentation is independent of apoptosis induction. HeLa cells stably expressing mitoDsRed GFP-Parkin were incubated with serum-free full medium or starvation medium and treated with $5 \mu \mathrm{M}$ arzanol or a combination of $4 \mu \mathrm{M}$ antimycin and $10 \mu \mathrm{M}$ oligomycin (A/O) with/without $10 \mu \mathrm{M}$ QVD for $6 \mathrm{~h}$. Shown blots are representative of three independent experiments. $\mathrm{L}$ and $\mathrm{S}$ indicate long and short forms of OPA1, respectively. Asterisk indicates an unspecific band.

\section{Discussion}

The modulation of autophagy is used as an anticancer treatment in more than eighty clinical trials treating several different cancer types (https://clinicaltrials.gov/; accessed on April 12, 2021). We used the top-down drug discovery approach to identify eight potential inducers and sixty-four potential inhibitors of autophagy. Among these compounds, we identified arzanol as a novel modulator of autophagy. Due to its strong effect on LC3-II accumulation and beneficial chemical characteristics like feasible compound isolation, commercial availability, and chemical stability, we selected it for a more detailed characterization.

In our assays, arzanol was initially identified as an inhibitor of starvation-induced autophagy by causing accumulation of overexpressed mCitrine-LC3 in murine fibroblasts. Consistently, it also caused the accumulation of overexpressed GFP-LC3 and endogenous total LC3 as well as lipidated LC3 in human cancer cells as determined by flow cytometry, fluorescence microscopy, and immunoblot analyses. Lipidation of LC3 is facilitated by the activation of the ubiquitin-like LC3 processing cascade involving ATG16 $\mathrm{L}^{46}$. Dudley et al. showed that ATG16L1 homodimerizes in order to bind to PI3Pcovered pre-autophagosomal sites, and that modulating the PI3P-binding capacity of ATG16L1 impairs lipidation of LC3 (ref. ${ }^{12}$ ). It is therefore not unlikely that the accumulation of ATG16L1 dots upon arzanol treatment under starvation conditions contributes to the increase in LC3-II observed in immunoblot analysis and to the increase in total LC3 measured by flow cytometry. As platforms for autophagosome biogenesis, ATG16L1positive dots are also likely to account for the number of small LC3-positive dots detected by microscopy. However, also the inhibition of the autophagic flux likely contributes to these phenomena by preventing 
A

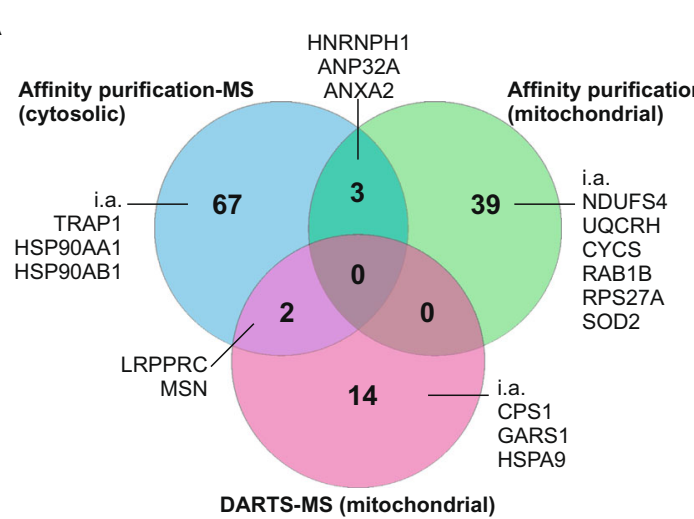

B
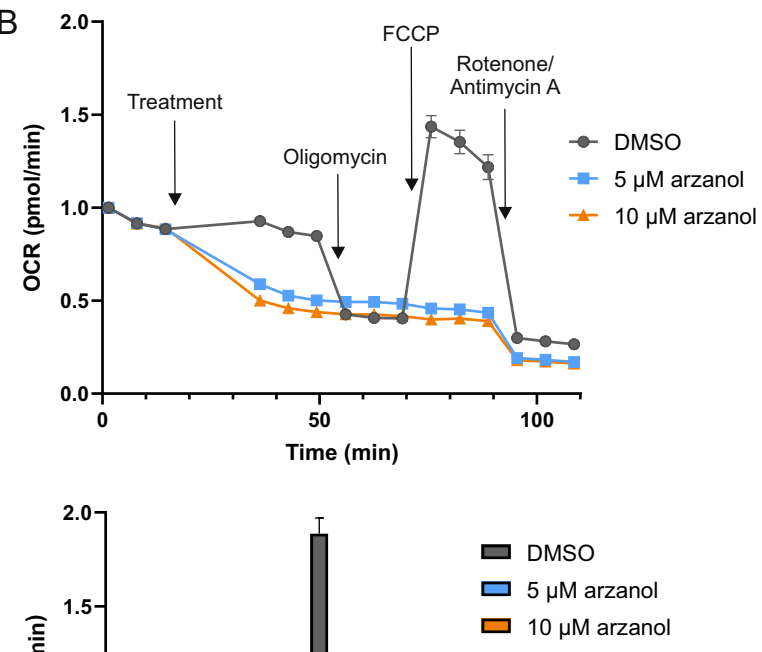

C

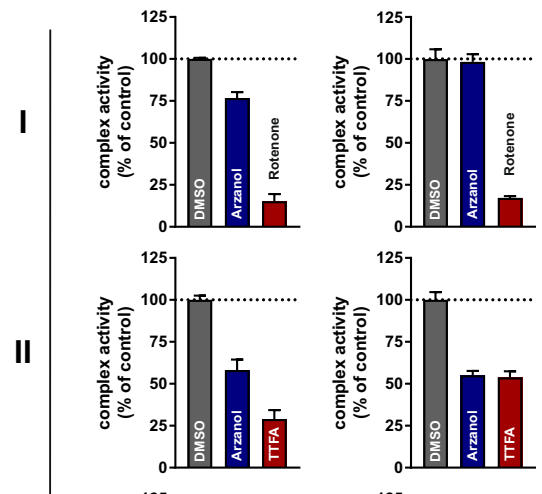

III
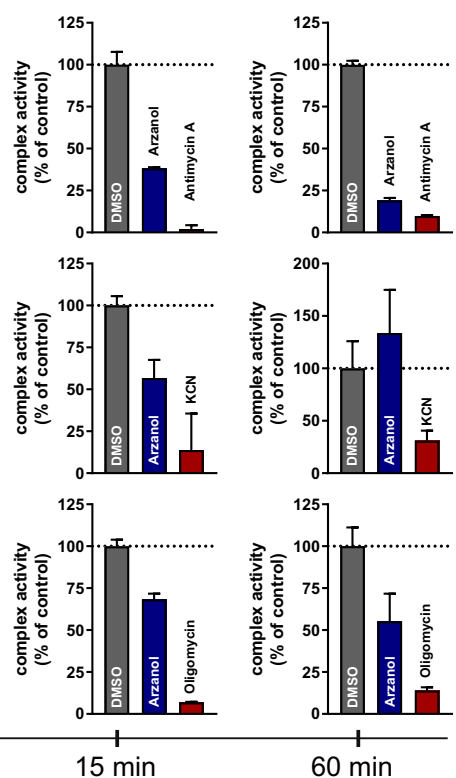

$E$

NQ01

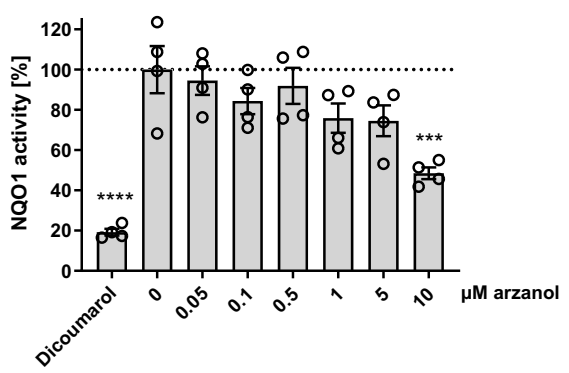

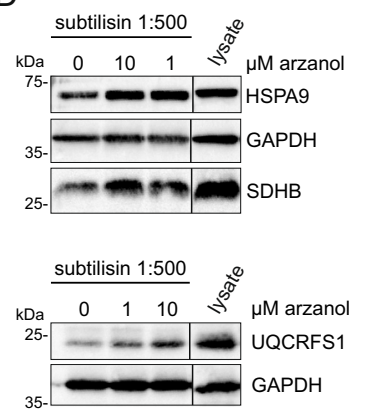

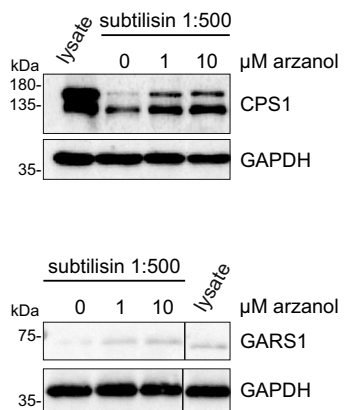

Fig. 6 (See legend on next page.) 
(see figure on previous page)

Fig. 6 Target identification for arzanol reveals OXPHOS complexes and possibly the $F_{1} F_{O}$ ATP synthase/complex $V$ as targets for arzanol. A Mass spectrometry (MS)-based target identification by affinity purification from HeLa cell whole lysates, and affinity purification and DARTS experiments from lysates of isolated mitochondria from HeLa cells reveal 125 novel potential targets of arzanol. B Cellular metabolism analysis was performed using a Seahorse Mito Stress Test Kit. Cells were incubated with arzanol for $15 \mathrm{~min}$ after measurement of the basal respiration and OCR was measured in at least octuplicates in three independent experiments. One representative experiment is shown (upper diagram). Respiration after treatment, maximal respiration, proton leak, and non-mitochondrial respiration were normalized to the basal respiration (lower diagram). Nonmitochondrial respiration $=$ minimum rate measurement after rotenone/antimycin $\mathrm{A}$ injection; basal respiration = last rate measurement before first injection minus non-mitochondrial respiration; maximal respiration = maximum rate measurement after FCCP injection minus non-mitochondrial respiration; proton leak = minimum rate measurement after oligomycin injection minus non-mitochondrial respiration. C Arzanol inhibits mainly OXPHOS complexes II and III in a respiratory chain activity assay of isolated mitochondria after incubation for 15 min and 60 min. Complex activities were measured using the MitoCheck kits in technical triplicates and normalized to the DMSO control. Bars show mean + SEM. D DARTS experiment validates selected OXPHOS complex proteins of complexes II and III. The lysate of isolated mitochondria from HeLa cells was incubated with 1 or $10 \mathrm{\mu M}$ arzanol and then subjected to subtilisin-dependent proteolytic cleavage. Arzanol stabilized SDHB, HSP9A, UQCRFS1, CPS1, and GARS1 against subtilisin treatment. Nonadjacent lanes (i.e., lysate control lane) are indicated by vertical black lines. Please note that in the SDHB/HSP9A panel the loading of the samples for 1 and $10 \mu \mathrm{M}$ arzanol treatment are exchanged. E Arzanol reduces the enzyme activity in an NQO1 activity assay similar to the positive control $20 \mu \mathrm{M}$ dicoumarol. Lysate from wild-type HeLa cells was subjected according to the manufacturer's manual. Bars show mean \pm SEM of biological quadruplets. Statistical analysis was performed using a one-way ANOVA with Dunnett's multiple comparison test comparing treated to untreated control samples; ${ }^{* * *} P<0.001,{ }^{* * * *} P<0.0001$.

autophagosome maturation and lysosomal LC3 degradation. While we observed smaller autophagosomes upon arzanol treatment, it also caused accumulation of p62. We interpret the pronounced accumulation of p62 upon arzanol treatment during starvation as an indicator of an inhibited autophagic flux. This is clearly supported by our flux analysis using the combination of arzanol and bafilomycin $\mathrm{A}_{1}$. It remains unclear whether the dysregulation in LC3 lipidation itself is ultimately sufficient to inhibit autophagy or if additional targets are involved.

Bansal et al. observed that ATG16L1 is able to colocalize with LC3-binding mitophagy receptors such as ubiquitin-binding optic neuropathy inducing protein

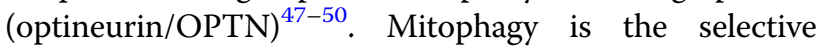
removal of damaged mitochondria and aims to deplete damaged organelles and their associated triggers such as mitotoxins ${ }^{43-45}$. To induce mitophagy, PINK accumulates at the outer membrane of damaged mitochondria where it recruits and activates the E3 ubiquitin ligase Parkin, which then ubiquitinates mitochondrial surface proteins. This allows the recruitment of autophagic receptors such as NDP52 and OPTN, but also ULK1, FIP200, ATG9A, WIPI proteins, and ATG16L1 to form a mature autophagosome around the damaged organelle ${ }^{51-53}$. When we checked for mitochondrial damage induced by arzanol during serum- and amino acid starvation, we observed the fragmentation of the mitochondria. We further found accumulation of PINK in immunoblot analysis and observed GFP-Parkin to run at a higher molecular mass, which we interpret as ubiquitinated Parkin. Along with the markers for mitochondrial damage, we detected cleaved OPA1, a protein required for mitochondrial fusion $^{54-57}$. In line with these data, we found arzanol to reduce the cellular OCR and the activity of mitochondrial OXPHOS complexes (mainly complexes II, III) and of complex V. Interestingly, among protein targets from affinity purification, we also identified NDUFS4, a protein of OXPHOS complex I. As novel targets for arzanol, we identified not only the complexes I, II, III and V, but also NQO1 and SOD2, which all function as quinonedependent reductases required for mitochondrial integrity $^{58-62}$. We hypothesize that arzanol binds to the quinone-binding pockets of these proteins and thus initiates the observed mitochondrial damage. TRAP1, another potential target of arzanol, regulates a metabolic switch between mitochondrial oxidative phosphorylation and glycolysis in cancer cells ${ }^{63}$. It is reported to maintain mitochondrial integrity downstream of OXPHOS complex I and PINK1 ${ }^{64}$. We speculate that arzanol-induced mitochondrial damage provokes autophagosome formation and ATG16L1 accumulation.

We tested arzanol in monotherapy and combinational therapy with CDDP against sensitive and CDDP-resistant bladder cancer cells. CDDP-based chemotherapy is the first-line treatment in many cases of advanced or metastatic urothelial carcinoma ${ }^{65}$. However, innate and acquired chemoresistance remain a main reason for cancer-related lethality in patients. Similar to other autophagy inhibitors that reduced tumor growth rate and prolonged patient survival ${ }^{66-70}$, we found that monotherapy with arzanol reduced the viability of both CDDPsensitive and -resistant bladder carcinoma cells while it sensitized the parental RT-112 cells to CDDP treatment in combination therapy. Clearly, in order to serve as lead compound for the development of anticancer drugs, the serum-binding properties of arzanol have to be addressed. We observed that arzanol was ineffective in the serumsupplied growth medium, thus preventing its systemic usage. Serum albumin has been suggested as a carrier for anticancer agents by prolonging the circulation half-life of 
drugs and thus promoting their accumulation within tumors $^{71}$. Although our work represents a preclinical study based on cellular model systems, we hypothesize that it might be a worthwhile objective to derivatize arzanol in such a way that it keeps its serum-binding properties while regaining its biological activity.

Conclusively, from our data we characterize arzanol as an inducer of mitochondrial damage and autophagosome formation and as an inhibitor of autophagy. Its different targets might account for the multifaceted mode of action that leads to cytotoxic effects in bladder cancer cells. Taking into consideration its pharmacological activities, we propose arzanol as a new lead structure for the treatment of bladder cancer and suggest further investigations on its target- and cancer-specificity.

\section{Materials and methods Reagents}

Natural compounds isolated from endophytic fungi, lichens, marine sponges, or plants were provided by Peter Proksch (Institute of Pharmaceutical Biology and Biotechnology of the Heinrich Heine University (Düsseldorf, Germany). Bafilomycin $\mathrm{A}_{1}$ (Sigma-Aldrich, \#B1793) and arzanol (Sigma-Aldrich, \#SBR00002) were dissolved in dimethyl sulfoxide (DMSO; AppliChem GmbH, \#A3672). We purchased antimycin A from Sigma (\#A8674) and oligomycin A from Toronto Research Chemicals (\#O532970). For transfection of cells, we used FuGENE ${ }^{\circledR}$ 6 (Promega, \#E2692) and polybrene (hexadimethrine bromide; Sigma-Aldrich, \#H9268-106). Immunoblots were performed using Immobilon ${ }^{\mathrm{TM}}$-FL PVDF membrane (Merck-Millipore, \#IPFL00010), milk powder (Carl Roth, \#T145.2), and Protease Inhibitor Cocktail powder (SigmaAldrich, \#P2714-1BTL). Cells were cultivated using full medium DMEM (Gibco ${ }^{\circledR}$ by Life Technologies, \#41965039), starvation medium EBSS (Gibco ${ }^{\circledR}$ by Life Technologies, \#24010-043), PBS (Gibco ${ }^{\circledR}$ by Life Technologies, \#14190-094), fetal bovine serum (GE Healthcare, \#A15-101), 0.05\% trypsin/EDTA solution (Gibco ${ }^{\circledR}$ by Life Technologies, \#25300-062), penicillin/streptomycin (10,000 U/ml, Biochrom GmbH, \#A2213), puromycin (InvivoGen, ant-pr), or blasticidin (InvivoGen, ant-bl).

For immunoblotting, antibodies against $\mathrm{ACTB} / \beta$-actin (Sigma-Aldrich, \#A5316), LC3 (Cell Signaling Technology, \#2775), SQSTM1/p62 (PROGEN Biotechnik, GP62C), ULK1 (clone D8H5, Cell Signaling Technology, \#8054), phospho ULK1 Ser758 (Cell Signaling Technology, \#6888), phospho TSC2 Ser939 (Cell Signaling Technology, \#3615), phospho p70S6K Ser371 (Cell Signaling Technology, \#9208), OPA1 (described previously $^{72}$ ), PINK (Cell Signaling Technology, \#6946), Parkin (Abcam, \#ab15954), SDHB (Thermo Fisher, \#459230), UQCRFS1 (Invitrogen, \#MA5-27471), CPS1 (Proteintech, \#65011-1-Ig), GAPDH (Invitrogen,
\#437000), HSPA9/GRP 75 (clone D-9, Santa Cruz Biotechnology, \#sc-133137) and GARS1/GlyRS (clone D-10, Santa Cruz Biotechnology, \#sc-365311) were used. IRDye 800 - or IRDye 680-conjugated secondary antibodies were purchased from LI-COR Biosciences (\#926-32210/11, \#926-68070/71, \#926-68024, \#926-68077, \#926-32214).

For immunofluorescence, antibodies against ATG16L (MBL \#PM040), LC3B (MBL \#PM036 and \#M152-3), WIPI2 (AbD Serotec/BIORAD, \#MCA5780GA), TOM20 (Santa Cruz Biotechnology, \#17764) were used. Alexa Fluor ${ }^{\circledR}$ 488-conjugated and Alexa Fluor ${ }^{\circledR}$ 647-conjugated antibodies were purchased from Jackson ImmunoResearch Laboratories (Alexa Fluor ${ }^{\circledR} 488$ AffiniPure Donkey Anti-Mouse IgG ( $\mathrm{H}+\mathrm{L})$, \#715-545-151; Alexa Fluor ${ }^{\circledR} 488$ AffiniPure Goat Anti-Rabbit IgG $(\mathrm{H}+\mathrm{L})$, \#111-545-003; Alexa Fluor ${ }^{\circledR} 647$ AffiniPure Goat AntiRabbit IgG (H+L), \#111-605-003; Alexa Fluor ${ }^{\circledR} 647$ AffiniPure Goat Anti-Mouse IgG (H + L), \#115-605-003).

NQO1 activity of HeLa cells was measured using the NQO1 Activity Assay Kit (Abcam, \#184867) according to the manufacturer. OXPHOS complex activities were measured from isolated mitochondria using the MitoCheck Complex Activity Assay Kits (Cayman Chemicals, \#700930, \#700940, \#700950, \#700990, \#701000). Both assays were measured using a microplate reader (BioTek, Synergy Mx). Cellular metabolism analysis was carried out using a Mito Stress Test Kit (Agilent Technologies, \# 103015-100); this kit includes oligomycin, FCCP, rotenone, and antimycin A.

\section{Generation and culture of cell lines}

Wild-type mouse embryonic fibroblasts (MEFs) (kindly provided by Tullia Lindsten, Memorial Sloan Kettering Cancer Center, New York City, USA) were retrovirally transfected with pMSCVblast/mCitrine-LC3B. Generation of pMSCVblast/mCitrine-LC3B was described previously $^{73}$. For transfection, Plat-E cells (kindly provided by Toshio Kitamura, Institute of Medical Science, University of Tokyo, Japan) were transfected with $1.9 \mu \mathrm{g}$ pMSCVbased retroviral vectors using FuGENE ${ }^{\circledR} 6$ (Promega) transfection reagent according to the manufacturer's manual. After $48 \mathrm{~h}$, retroviral supernatant was collected and used for the infection of MEFs in combination with $9 \mu \mathrm{g} / \mathrm{mL}$ polybrene (Sigma-Aldrich; H9268-106). The cells were incubated for 3 days prior to selection with $35 \mu \mathrm{g} / \mathrm{ml}$ blasticidin.

Wild-type HeLa cells (kindly provided by Richard Youle, John Edward Porter Neuroscience Research Center, Bethesda, USA) were retrovirally transfected with pMRXIP/GFP-LC3-RFP-LC3 $\Delta$ G (kindly provided by Noboru Mizushima, Department of Biochemistry and Molecular Biology, University of Tokyo, Tokyo, Japan; Addgene plasmid \#84572; http://n2t.net/addgene:84572; RRID: Addgene_84572). Alternatively, mito-DsRed-expressing 
HeLa cells (kindly provided by Aviva M. Tolkovsky, Department of Clinical Neurosciences, University of Cambridge, Cambridge, United Kingdom) were retrovirally transfected with pMSCVpuro/EGFP-Parkin. This plasmid was generated by inserting EGFP cDNA and human Parkin cDNA into pMSCVpuro. For retroviral transfection of HeLa cells, Plat-E cells were transfected with $1.9 \mu \mathrm{g}$ pMRX- or pMSCVpuro-based retroviral vectors and $1.0 \mu \mathrm{g}$ pVSV-G vector DNA using FuGENE ${ }^{\circledR} 6$ (Promega) transfection reagent according to the manufacturer's manual. After $48 \mathrm{~h}$, retroviral supernatant was collected and used for the infection of HeLa cells in combination with $9 \mu \mathrm{g} / \mathrm{mL}$ polybrene (Sigma-Aldrich; H9268-106). The cells were incubated for three days prior to selection with $2.5 \mu \mathrm{g} / \mathrm{ml}$ puromycin. For Fig. 2J, cells were subcloned in order to isolate clones that properly express GFP-LC3-RFP-LC3 $\Delta$ G, since homologous recombination can occur between the two LC3 sequences during transfection ${ }^{35}$.

All cell lines including urothelial bladder carcinoma cells RT-112 and their CDDP-resistant equivalent cell line (previously described ${ }^{74}$ and kindly provided by Margaretha Skowron, Department of Urology, Medical Faculty, Heinrich Heine University, Düsseldorf, Germany) were cultured in high glucose (4.5 g/l) DMEM supplemented with 10\% FCS at $37{ }^{\circ} \mathrm{C}$ in a $5 \% \mathrm{CO}_{2}$ humidified atmosphere. For CDDP-resistance, RT-112 cells were kept at $12 \mu \mathrm{g} / \mathrm{ml}$ CDDP. For amino acid starvation, cells were washed once with PBS and incubated for the indicated times in EBSS.

\section{High-throughput autophagy screening}

Mouse embryonic fibroblasts stably expressing mCitrine-LC3 were incubated with $10 \mu \mathrm{M}$ of each natural compound solved in DMSO in serum-containing full medium or starvation medium for $6 \mathrm{~h}$. During screening, compound samples were blinded by labeling with a randomized code and unblinded post-experiments. Cells were harvested by trypsinization, washed using PBS and centrifugation at $300 \times g$, and measured via flow cytometry of 10,000 events in the FITC channel of an LSRFortessa (Becton Dickinson, Heidelberg, Germany). Median fluorescence intensities were measured in biological triplicates and normalized to the DMSO control.

\section{GFP-LC3-RFP-LC3 $\Delta$ G assay}

HeLa cells stably expressing GFP-LC3-RFP-LC3 $\Delta$ G were incubated with the indicated concentrations of selfisolated or commercial arzanol solved in DMSO in the starvation medium for $6 \mathrm{~h}$. Cells were harvested and analyzed using flow cytometry as described above.

\section{Immunoblotting}

Cleared cell lysates were prepared and subjected to immunoblotting as described before ${ }^{73}$. Signal intensities of protein bands were quantified using Image Studio Lite 4.0 (LI-COR), and each band was normalized to the average protein signal to correct for technical variance. The signals were then normalized to the corresponding loading control (ACTB). Panels for at least three biological replicates were prepared using GraphPad Prism 7.0.

\section{Fluorescence microscopy}

On the day before treatment, cells were grown on glass coverslips (Marienfeld). After treatment, cells were fixed with $4 \%$ formaldehyde-PBS for $30 \mathrm{~min}$ on ice and quenched with $50 \mathrm{mM} \mathrm{NH} \mathrm{NH}_{4} \mathrm{Cl}$ for $15 \mathrm{~min}$. For immunofluorescence labeling, cells were then permeabilized with $0.2 \%$ Triton X-100-PBS for $15 \mathrm{~min}$, or $50 \mu \mathrm{g} / \mathrm{ml}$ digitonin (Roth, \#4005) for 5 min according to the antibody manufacturers. Samples were blocked with 3\% BSA (Roth, \#8076)-PBS for $30 \mathrm{~min}$ and incubated with primary antibodies for 1-2 h. After washing and $30 \mathrm{~min}$ of secondary antibody incubation, samples were again washed three times with PBS. Cells were embedded in ProLong Glass Antifade Mountant (Thermo Fisher Scientific, \#P36980), including DAPI. Imaging was performed with a Zeiss Axio Observer 7 fluorescence microscope (Zeiss, Köln, Germany) with a Plan Apochromat 40x/1.4 oil objective (Zeiss, Köln, Germany). Quantification of images was performed with ImageJ. For that, signals and nuclei were counted per image and a signal-to-nuclei ratio was calculated. Macros for the quantifications are provided in Supplementary Methods.

\section{Cell viability assays}

Cell viability was determined using the colorimetric Alamar Blue and MTT assays, which measure the reduction of non-fluorescent dyes to the fluorescent metabolites resorufin and formazan, respectively. For both assays, cells were cultivated in 96-well plates. The following day, the cells were treated with cisplatin and/or arzanol for $24 \mathrm{~h}$. For the MTT assay, MTT (Roth \#4022) was added to the cells at $0.5 \mathrm{mg} / \mathrm{ml}$ concentration and incubated at $37^{\circ} \mathrm{C}$ for $1 \mathrm{~h}$. Afterward, the plates were centrifuged at $600 \times g$ and $4{ }^{\circ} \mathrm{C}$ for $5 \mathrm{~min}$, and cells were lysed in DMSO for $20 \mathrm{~min}$ in the dark. Finally, the absorbance was measured at $570 \mathrm{~nm}$ and $650 \mathrm{~nm}$ for reference, using a microplate reader (BioTek, Synergy $\mathrm{Mx})$. For the Alamar blue assay, $40 \mu \mathrm{M}$ resazurin sodium salt (Cayman Chemicals, \#14322) was added to the cells and incubated at $37^{\circ} \mathrm{C}$ for $3 \mathrm{~h}$. Afterward, the absorbance was measured at $590 \mathrm{~nm}$, using a microplate reader (BioTek, Synergy Mx). The mean of the absorbance of untreated control samples was set as $100 \%$.

\section{Cellular metabolism analysis}

For cellular metabolism analysis, a Mito Stress Test Kit (Agilent Technologies, Santa Clara, CA, USA) was applied 
according to the manufacturer's instructions using a Seahorse XFe96 Extracellular Flux Analyzer (Agilent Technologies). The FCCP concentration of $2.5 \mu \mathrm{M}$ and the cell density of 15,000 cells/well were titrated prior to the experiments. Treatment with arzanol was performed after measurement of basal respiration. Basal respiration can be defined as respiration before the first injection by the Seahorse system. The maximal respiration was defined as the OCR after FCCP injection minus the OCR after blocking the mitochondrial respiration after rotenone and antimycin A injection (non-mitochondrial OCR). Proton leak is defined as the minimum rate measurement after oligomycin injection minus the non-mitochondrial respiration.

\section{Isolation of mitochondria}

For isolation of mitochondria, wild-type HeLa cells were cultivated on $150-\mathrm{mm}$ diameter tissue culture-treated dishes (Sarstedt) and $\sim 3.6 \times 10^{8}$ cells were harvested the next day via scraping. Cells were pelleted at $500 \times g$ for 5 min and washed twice with PBS (Gibco). The pellet was resuspended in $10 \mathrm{ml}$ mitochondria isolation buffer (210 mM mannitol, $70 \mathrm{mM}$ sucrose, $1 \mathrm{mM}$ EDTA, $20 \mathrm{mM}$ HEPES, and protease inhibitor cocktail (Sigma-Aldrich, \#P2714)) for $5 \mathrm{~min}$ on ice before rupturing by seven strokes through a $26 \mathrm{G}$ cannula. The cell lysate was then centrifuged at $1000 \times g$ and $4{ }^{\circ} \mathrm{C}$ for $5 \mathrm{~min}$ and the supernatant was collected. The remaining pellet of nonlysed cells was resuspended in $2 \mathrm{ml}$ of mitochondria isolation buffer and ruptured again before centrifugation, and the two fractions were pooled. The pooled lysate was centrifuged again at $1000 \times g$ and $4{ }^{\circ} \mathrm{C}$ for $5 \mathrm{~min}$, and the pellet was discarded. The remaining lysate was centrifuged at $8000 \times g$ and $4{ }^{\circ} \mathrm{C}$ for $10 \mathrm{~min}$. The supernatant (cytosolic fraction) was collected and centrifuged again before transferring into a new tube and freezing in liquid nitrogen. The pellet (mitochondrial fraction) was washed three times at $8000 \times g$ for $10 \mathrm{~min}$ in $250 \mu \mathrm{l}$ of mitochondria isolation buffer. The pellet was finally centrifuged at $10,000 \times g$ and $4{ }^{\circ} \mathrm{C}$ for $10 \mathrm{~min}$. The supernatant was discarded and the pellet containing isolated mitochondria was frozen in liquid nitrogen and stored at $-80^{\circ} \mathrm{C}$.

\section{Preparation of cellular and mitochondrial HeLa lysates}

Cellular HeLa lysates were obtained by mechanical lysis in PBS pH 7.4 (137 mM NaCl, 2,7 mM KCl, $10 \mathrm{mM}$ $\mathrm{Na}_{2} \mathrm{HPO}_{4}, 2 \mathrm{mM} \mathrm{KH}_{2} \mathrm{PO}_{4}$ ) containing 0.1\% IGEPAL CA-630 and a protease inhibitor cocktail (SigmaAldrich). Mitochondria were lysed in a buffer composed of $1.5 \%$ digitonin, a protease inhibitor cocktail (SigmaAldrich), $150 \mathrm{mM} \mathrm{NaCl}, 10 \mathrm{mM}$ Tris/ $\mathrm{HCl}$ (pH 7.5), and $5 \mathrm{mM}$ EDTA. After $15 \mathrm{~min}$ at $4{ }^{\circ} \mathrm{C}$, debris was discarded by centrifugation at $20,000 \times g\left(30 \mathrm{~min}\right.$ at $\left.4^{\circ} \mathrm{C}\right)$.
The protein concentration of the obtained supernatants was determined by Bradford spectrophotometric assay (BioRad Laboratories, Hercules, CA) and adjusted to $3 \mathrm{mg} / \mathrm{ml}$.

\section{Affinity purification from lysates and isolated mitochondria}

Arzanol bearing resin has been obtained as reported by Del Gaudio et al. $^{75}$. The arzanol-containing and the control resin were separately incubated with a solution containing $800 \mu \mathrm{g}$ of proteins for $1 \mathrm{~h}$ under stirring at $4{ }^{\circ} \mathrm{C}$. After the incubation period, unspecifically adsorbed proteins were removed performing three rounds of washing with PBS, whereas bound proteins were eluted with $50 \mu \mathrm{l}$ of $100 \mathrm{mM}$ Tris (pH 6.8), $4 \%$ (v/v) sodium dodecyl sulfate (SDS), 0.2\% (v/v) Blue Bromophenol, 20\% (v/v) glycerol, and $2 \% \beta$-mercaptoethanol buffer. Each resin was then boiled at $95^{\circ} \mathrm{C}$ for $5 \mathrm{~min}$ and $15 \mu \mathrm{l}$ of the obtained eluates were subjected to $1 \mathrm{D}-\mathrm{SDS}-\mathrm{PAGE}$ (12\% polyacrylamide). The resulting gel was then treated with $40 \% \mathrm{MeOH}, 10 \%$ $\mathrm{CH}_{3} \mathrm{COOH}$, and $50 \% \quad \mathrm{H}_{2} \mathrm{O}$ and stained by Coomassie Blue.

\section{Drug affinity responsive target stability}

In total, $300 \mu \mathrm{g}$ protein aliquots were either incubated with DMSO (vehicle control) or with arzanol (1 and $10 \mu \mathrm{M}$ final concentrations) for $1 \mathrm{~h}$ at room temperature and under agitation. The obtained samples were then treated with the unspecific protease subtilisin (SigmaAldrich, \#P5380) (enzyme to proteins ratio of 1:500 w/w) and left shaking for $30 \mathrm{~min}$ at $25^{\circ} \mathrm{C}$. Proteolysis was then quenched by adding PMSF (phenylmethylsulfonyl fluoride, Sigma-Aldrich, \#P7626, $1 \mathrm{mM}$ final concentration) to each sample. Subsequently, all of the samples were prepared for 1D-SDS-PAGE and $20 \mu \mathrm{g}$ were loaded on a 4-12\% Bis-Tris Criterion ${ }^{\text {TM }}$ XT Precast Gel (BioRad Laboratories, \#3450123), which was then stained with a Coomassie solution. The experiment was carried out in duplicates.

\section{In situ gel digestion}

Protein bands were excised from the gels and submitted to an in situ tryptic digestion protocol ${ }^{76}$. Briefly, gel slices were reduced by $6.5 \mathrm{mM}$ 1,4-dithiothreitol (DTT) and alkylated by $54 \mathrm{mM}$ iodoacetamide. A $12 \mathrm{ng} / \mu \mathrm{l}$ trypsin/ LysC solution (Promega, Madison, Wisconsin) was used to digest proteins. The enzyme excess was then discarded and replaced with ammonium bicarbonate (AmBic, $40 \mu \mathrm{l}$, $50 \mathrm{mM}, \mathrm{pH} 8.5$ ), allowing protein digestion to carry on overnight at $37^{\circ} \mathrm{C}$. Subsequently, supernatants were collected and peptides were extracted from each gel slice using $100 \% \mathrm{CH}_{3} \mathrm{CN}$. The obtained peptide mixtures were dried in vacuo and dissolved in formic acid (FA, 10\%) for LC-MS/MS analysis. 


\section{Liquid chromatography and mass spectrometry analysis (LC-MS/MS)}

In total, $5 \mu \mathrm{l}$ of each sample were injected into a nanoACQUITY UPLC system (Waters, Milford, MA, USA), equipped with a 1.7- $\mu \mathrm{m}$ BEH C18 column (Waters). Peptide elution was achieved with a linear gradient of mobile phase B from 20 to $90 \%$ in 65 min (mobile phase A: $95 \% \mathrm{H}_{2} \mathrm{O}, 5 \% \mathrm{CH}_{3} \mathrm{CN}, 0.1 \%$ acetic acid; mobile phase B: $95 \% \mathrm{CH}_{3} \mathrm{CN}, 5 \% \mathrm{H}_{2} \mathrm{O}, 0.1 \%$ acetic acid) at a flow rate of $280 \mathrm{nl} / \mathrm{min}$. MS and MS/MS data were acquired on an LTQ Orbitrap XL high-performance liquid chromatography MS system (Thermo-Scientific, Waltham, MA, USA), interfaced with a nanoESI source. The ten most intense doubly and triply charged peptide ions were fragmented. MS data were then processed by the MS Converter General User Interface software (ProteoWizard; http://proteowizard.sourceforge.net/project.html) and submitted to MASCOT Deamon (version 5.1, Matrix Science, London, UK) for protein identification, employing the SwissProt database (release November 2019, 561,344 entries) and the following settings: two missed cleavages; carbamidomethyl (C) as fixed modification; oxidation (M) and phosphorylation (ST) as variable modifications; peptide tolerance $30 \mathrm{ppm}$; MS/MS tolerance $0.8 \mathrm{Da}$.

\section{Statistics}

Sample sizes were chosen with reference to pragmatic considerations and the principle of saturation due to previously published work in this field and preliminary experiments. For high-throughput analysis of the flow cytometric mCitrine-LC3-based autophagy screening, we used uniformly minimal variance unbiased estimate (UMVUE) of strictly standardized mean difference $(\mathrm{SSMD})^{30}$. For the quantifications of the immunoblots shown in Fig. 2, statistical analysis was performed using a two-way ANOVA with Dunnett's multiple comparison test comparing treated to untreated control samples. For comparing size and number of dots in Fig. 3, statistical analysis was performed using an ordinary 1way ANOVA with Tukey's multiple comparison test comparing all treatments with each other. All $\mathrm{IC}_{50}$ values in Fig. 4 were calculated using GraphPad Prism 7.01 (function log (inhibitor) vs. response - variable slope (four parameters)). For NQO1 activity data (Fig. 6E), statistical analysis was performed using a one-way ANOVA with Dunnett's multiple comparison test comparing treated to untreated control samples. Representative data or means of biological replicates are shown for every experiment with error bars that indicate standard error. Numbers of technical or biological replicates and definition of $P$ values are indicated in the corresponding figure legend. All statistical analyses were performed using Prism v7.01 (GraphPad Software, La Jolla, CA, USA).

\begin{abstract}
Acknowledgements
We thank Aviva M. Tolkovsky (Department of Clinical Neurosciences, University of Cambridge, Cambridge, UK) for providing mito-DsRed-expressing HeLa cells. We thank Margaretha Skowron (Department of Urology, Medical Faculty, Heinrich Heine University, Düsseldorf, Germany) for providing RT-112 bladder carcinoma cells. We thank Julia Werner (Institute of Pharmaceutical Biology and Biotechnology, Faculty of Mathematics and Natural Sciences, Heinrich Heine University, Düsseldorf, Germany) for isolating arzanol used in Figs. 1 and 2. We thank Tullia Lindsten (Memorial Sloan Kettering Cancer Center, New York City, USA) for providing wild-type mouse embryonic fibroblasts. We thank Richard Youle (John Edward Porter Neuroscience Research Center, Bethesda, USA) for providing wild-type HeLa cells, We thank Toshio Kitamura (Institute of Medical Science, University of Tokyo, Japan) for providing Plat-E cells.
\end{abstract}

\section{Author details}

${ }^{1}$ Institute of Molecular Medicine I, Medical Faculty and University Hospital Düsseldorf, Heinrich-Heine-University Düsseldorf, Universitätsstraße 1, 40225 Düsseldorf, Germany. ${ }^{2}$ Department of Pharmacy, University of Salerno, Via Giovanni Paolo II 132, 84084 Salerno, Fisciano, Italy. ${ }^{3}$ Institute of Biochemistry and Molecular Biology I, Medical Faculty and University Hospital Düsseldorf, Heinrich-Heine-University Düsseldorf, Universitätsstraße 1, 40225 Düsseldorf, Germany. ${ }^{4}$ Institute of Pharmaceutical Biology and Biotechnology, Faculty of Mathematics and Natural Sciences, Heinrich-Heine-University Düsseldorf, Universitätsstraße 1, 40225 Düsseldorf, Germany

\section{Author contributions}

J.D. designed the experiments, performed flow cytometry analyses, microscopy, and cell viability assays, performed the NQO1 activity assay and immunoblot analyses, and prepared samples for AP-MS and DARTS. J.D. analyzed and interpreted the data and wrote the manuscript. L.B. performed cell viability assays, microscopy, and cellular metabolism analyses. F.S. performed the MitoCheck experiments. S.C. and M.C.M. performed DARTS and AP-MS. D.S. provided expertise on microscopy and quantifications. R.A. and A.S. R. performed and interpreted cellular metabolism analyses. P.B. generated EGFP-Parkin- and mito-DsRed-expressing HeLa cells. M.J.M., A.F., W.W., Y.S., N.B., and S.S. gave technical support. P.P. provided an in-house natural compound library. P.P. and B.S. supervised the project. All authors discussed the results and commented on the manuscript.

\section{Funding}

This study was supported by the Deutsche Forschungsgemeinschaft GRK 2158 (to P.P. and to B.S.), STO 864/4-3 (to B.S.), STO 864/5-1 (to B.S.), STO 864/6-1 (to B.S.), SFB1208-project B12 (to A.S.R.), and the Düsseldorf School of Oncology (to B.S.; funded by the Comprehensive Cancer Center Düsseldorf/Deutsche Krebshilfe and the Medical Faculty of the Heinrich Heine University Düsseldorf). S.C. and M.C.M. were supported by POR CAMPANIA FESR 2014/2020 Asse 1Obiettivo specifico 1.2-Azione 1.2. Progetto: Campania OncoTerapie CUP: B61G18000470007. Open Access funding enabled and organized by Projekt DEAL.

\section{Data availability}

The datasets used and/or analyzed during this study are available from the corresponding author on reasonable request.

Ethics statement

The authors declare that this study did not require ethical approval.

Conflict of interest

The authors declare no competing interests.

\section{Publisher's note}

Springer Nature remains neutral with regard to jurisdictional claims in published maps and institutional affiliations.

Supplementary information The online version contains supplementary material available at https://doi.org/10.1038/s41419-021-03830-5. 
Received: 15 December 2020 Revised: 5 May 2021 Accepted: 10 May 2021 Published online: 31 May 2021

\section{References}

1. Choi, H. et al. NPCARE: database of natural products and fractional extracts for cancer regulation. J. Cheminform. 9, 2 (2017).

2. Newman, D. J. \& Cragg, G. M. Natural products as sources of new drugs from 1981 to 2014. J. Nat. Prod. 79, 629-661 (2016).

3. Baker, D. D., Chu, M., Oza, U. \& Rajgarhia, V. The value of natural products to future pharmaceutical discovery. Nat. Prod. Rep. 24, 1225-1244 (2007).

4. Frake, R. A., Ricketts, T., Menzies, F. M. \& Rubinsztein, D. C. Autophagy and neurodegeneration. J. Clin. Investig. 125, 65-74 (2015).

5. Harris, H. \& Rubinsztein, D. C. Control of autophagy as a therapy for neurodegenerative disease. Nat. Rev. Neurol. 8, 108-117 (2011).

6. Levine, B. \& Kroemer, G. Biological functions of autophagy genes: a disease perspective. Cell 176, 11-42 (2019).

7. Mizushima, N., Levine, B., Cuervo, A. M. \& Klionsky, D. J. Autophagy fights disease through cellular self-digestion. Nature 451, 1069-1075 (2008).

8. Graef, M. \& Nunnari, J. Mitochondria regulate autophagy by conserved signalling pathways. EMBO J. 30, 2101-2114 (2011).

9. Li, L., Chen, Y. \& Gibson, S. B. Starvation-induced autophagy is regulated by mitochondrial reactive oxygen species leading to AMPK activation. Cell. Signal. 25, 50-65 (2013).

10. Papandreou, I., Lim, A. L., Laderoute, K. \& Denko, N. C. Hypoxia signals autophagy in tumor cells via AMPK activity, independent of HIF-1, BNIP3, and BNIP3L. Cell Death Differ. 15, 1572-1581 (2008).

11. Axe, E. L. et al. Autophagosome formation from membrane compartments enriched in phosphatidylinositol 3-phosphate and dynamically connected to the endoplasmic reticulum. J. Cell Biol. 182, 685-701 (2008).

12. Dudley, L. J. et al. Intrinsic lipid binding activity of ATG16L1 supports efficient membrane anchoring and autophagy. EMBO J. 38, e100554 (2019).

13. Lee, Y. \& Weihl, C. C. Regulation of SQSTM1/p62 via UBA domain ubiquitination and its role in disease. Autophagy 13, 1615-1616 (2017).

14. Degenhardt, K. et al. Autophagy promotes tumor cell survival and restricts necrosis, inflammation, and tumorigenesis. Cancer Cell 10, 51-64 (2006).

15. Martinez-Outschoorn, U. E. et al. Oxidative stress in cancer associated fibroblasts drives tumor-stroma co-evolution: A new paradigm for understanding tumor metabolism, the field effect and genomic instability in cancer cells. Cell Cycle 9, 3256-3276 (2010).

16. Mathew, R., Karantza-Wadsworth, V. \& White, E. Assessing metabolic stress and autophagy status in epithelial tumors. Methods Enzymol. 453, 53-81 (2009).

17. New, J. et al. Secretory autophagy in cancer-associated fibroblasts promotes head and neck cancer progression and offers a novel therapeutic target. Cancer Res. 77, 6679-6691 (2017).

18. Rouschop, K. M. et al. The unfolded protein response protects human tumor cells during hypoxia through regulation of the autophagy genes MAP1LC3B and ATG5. J. Clin. Investig. 120, 127-141 (2010).

19. Karantza-Wadsworth, $\mathrm{V}$. et al. Autophagy mitigates metabolic stress and genome damage in mammary tumorigenesis. Genes Dev. 21, 1621-1635 (2007).

20. Lazova, R. et al. Punctate LC3B expression is a common feature of solid tumors and associated with proliferation, metastasis, and poor outcome. Clin. Cancer Res. 18, 370-379 (2012).

21. Wiedmer, T. et al. Autophagy inhibition improves sunitinib efficacy in pancreatic neuroendocrine tumors via a lysosome-dependent mechanism. Mol. Cancer Ther. 16, 2502-2515 (2017).

22. Cook, K. L. et al. Hydroxychloroquine inhibits autophagy to potentiate antiestrogen responsiveness in ER+ breast cancer. Clin. Cancer Res. 20, 3222-3232 (2014).

23. Degtyarev, M. et al. Akt inhibition promotes autophagy and sensitizes PTENnull tumors to lysosomotropic agents. J. Cell Biol. 183, 101-116 (2008).

24. Ratikan, J. A., Sayre, J. W. \& Schaue, D. Chloroquine engages the immune system to eradicate irradiated breast tumors in mice. Int. J. Radiat. Oncol. Biol. Phys. 87, 761-768 (2013).

25. Chen, J. L. et al. Autophagy induction results in enhanced anoikis resistance in models of peritoneal disease. Mol. Cancer Res. 15, 26-34 (2017).

26. Cui, L. et al. Radiation induces autophagic cell death via the p53/DRAM signaling pathway in breast cancer cells. Oncol. Rep. 35, 3639-3647 (2016).

27. Kim, D. E. et al. Raloxifene induces autophagy-dependent cell death in breast cancer cells via the activation of AMP-activated protein kinase. Mol. Cells $\mathbf{3 8}$ 138-144 (2015).
28. Peng, P. L., Kuo, W. H., Tseng, H. C. \& Chou, F. P. Synergistic tumor-killing effect of radiation and berberine combined treatment in lung cancer: the contribution of autophagic cell death. Int. J. Radiat. Oncol. Biol. Phys. 70, 529-542 (2008).

29. Yu, P. et al. Anti-proliferation of triple-negative breast cancer cells with physagulide P: ROS/JNK signaling pathway induces apoptosis and autophagic cell death. Oncotarget 8, 64032-64049 (2017)

30. Zhang, X. D. Illustration of SSMD, z score, SSMD*, $z^{*}$ score, and t statistic for hit selection in RNAi high-throughput screens. J. Biomol. Screen. 16, 775-785 (2011).

31. Werner, J. et al. Pyrone derivatives from Helichrysum italicum. Fitoterapia 133 80-84 (2019).

32. Appendino, G. et al. Arzanol, an anti-inflammatory and anti-HIV-1 phloroglucinol alpha-Pyrone from Helichrysum italicum ssp. microphyllum. J. Nat. Prod. 70, 608-612 (2007)

33. Rosa, A., Atzeri, A., Nieddu, M. \& Appendino, G. New insights into the antioxidant activity and cytotoxicity of arzanol and effect of methylation on its biological properties. Chem. Phys. Lipids 205, 55-64 (2017).

34. Mizushima, N. \& Yoshimori, T. How to interpret LC3 immunoblotting. Autophagy 3, 542-545 (2007).

35. Kaizuka, T. et al. An autophagic flux probe that releases an internal control. Mol. Cell 64, 835-849 (2016).

36. Klionsky, D. J. et al. Guidelines for the use and interpretation of assays for monitoring autophagy. Autophagy 12, 1-222 (2016).

37. Dooley, H. C. et al. WIPI2 links LC3 conjugation with PI3P, autophagosome formation, and pathogen clearance by recruiting Atg12-5-16L1. Mol. Cell 55, 238-252 (2014).

38. Polson, H. E. et al. Mammalian Atg18 (WIPI2) localizes to omegasomeanchored phagophores and positively regulates LC3 lipidation. Autophagy 6 506-522 (2010)

39. Quan, Y. et al. Inhibition of autophagy enhances the anticancer effect of enzalutamide on bladder cancer. Biomed. Pharmacother. 120, 109490 (2019).

40. Schlütermann, D. et al. Targeting urothelial carcinoma cells by combining cisplatin with a specific inhibitor of the autophagy-inducing class III Ptdlns3K complex. Urol. Oncol. 36, 160.e161-160.e113 (2018).

41. White, E. \& DiPaola, R. S. The double-edged sword of autophagy modulation in cancer. Clin. Cancer Res. 15, 5308-5316 (2009).

42. Hamid, R., Rotshteyn, Y., Rabadi, L., Parikh, R. \& Bullock, P. Comparison of Alamar blue and MTT assays for high through-put screening. Toxicol. Vitr. 18, 703-710 (2004).

43. Vives-Bauza, C. et al. PINK1-dependent recruitment of Parkin to mitochondria in mitophagy. Proc. Natl Acad. Sci. USA 107, 378-383 (2010).

44. Tanaka, A. et al. Proteasome and p97 mediate mitophagy and degradation of mitofusins induced by Parkin. J. Cell Biol. 191, 1367-1380 (2010).

45. Twig, G. \& Shirihai, O. S. The interplay between mitochondrial dynamics and mitophagy. Antioxid. Redox Signal 14, 1939-1951 (2011).

46. Fujita, N. et al. The Atg16L complex specifies the site of LC3 lipidation for membrane biogenesis in autophagy. Mol. Biol. Cell 19, 2092-2100 (2008).

47. Wong, Y. C. \& Holzbaur, E. L. Temporal dynamics of PARK2/parkin and OPTN/ optineurin recruitment during the mitophagy of damaged mitochondria. Autophagy 11, 422-424 (2015).

48. Bansal, M. et al. Optineurin promotes autophagosome formation by recruiting the autophagy-related Atg12-5-16L1 complex to phagophores containing the Wipi2 protein. J. Biol. Chem. 293, 132-147 (2018).

49. Moore, A. S. \& Holzbaur, E. L. Dynamic recruitment and activation of ALS associated TBK1 with its target optineurin are required for efficient mitophagy. Proc. Natl Acad. Sci. USA 113, E3349-E3358 (2016).

50. Wild, P. et al. Phosphorylation of the autophagy receptor optineurin restricts Salmonella growth. Science 333, 228-233 (2011).

51. Itakura, E., Kishi-Itakura, C., Koyama-Honda, I. \& Mizushima, N. Structures containing Atg9A and the ULK1 complex independently target depolarized mitochondria at initial stages of Parkin-mediated mitophagy. J. Cell Sci. 125 1488-1499 (2012).

52. Heo, J. M., Ordureau, A., Paulo, J. A., Rinehart, J. \& Harper, J. W. The PINK1PARKIN mitochondrial ubiquitylation pathway drives a program of OPTN/ NDP52 recruitment and TBK1 activation to promote mitophagy. Mol. Cell 60 7-20 (2015).

53. Lazarou, M. et al. The ubiquitin kinase PINK1 recruits autophagy receptors to induce mitophagy. Nature 524, 309-314 (2015).

54. Duvezin-Caubet, S. et al. Proteolytic processing of OPA1 links mitochondrial dysfunction to alterations in mitochondrial morphology. J. Biol. Chem. $\mathbf{2 8 1}$ 37972-37979 (2006) 
55. Baricault, L. et al. OPA1 cleavage depends on decreased mitochondrial ATP level and bivalent metals. Exp. Cell Res. 313, 3800-3808 (2007).

56. Guillery, O. et al. Metalloprotease-mediated OPA1 processing is modulated by the mitochondrial membrane potential. Biol. Cell. 100, 315-325 (2008).

57. Ishihara, N., Fujita, Y., Oka, T. \& Mihara, K. Regulation of mitochondrial morphology through proteolytic cleavage of OPA1. EMBO J. 25, 2966-2977 (2006)

58. Benit, P., Lebon, S. \& Rustin, P. Respiratory-chain diseases related to complex III deficiency. Biochim. Biophys. Acta 1793, 181-185 (2009).

59. Byun, H. O., Kim, H. Y., Lim, J. J., Seo, Y. H. \& Yoon, G. Mitochondrial dysfunction by complex II inhibition delays overall cell cycle progression via reactive oxygen species production. J. Cell. Biochem. 104, 1747-1759 (2008).

60. Kwon, J. et al. Assurance of mitochondrial integrity and mammalian longevity by the p62-Keap1-Nrf2-Nqo1 cascade. EMBO R. 13, 150-156 (2012).

61. Li, Y. et al. Dilated cardiomyopathy and neonatal lethality in mutant mice lacking manganese superoxide dismutase. Nat. Genet. 11, 376-381 (1995).

62. Voets, A. M. et al. Transcriptional changes in OXPHOS complex I deficiency are related to anti-oxidant pathways and could explain the disturbed calcium homeostasis. Biochim. Biophys. Acta 1822, 1161-1168 (2012).

63. Yoshida, S. et al. Molecular chaperone TRAP1 regulates a metabolic switch between mitochondrial respiration and aerobic glycolysis. Proc. Natl Acad. Sci. USA 110, E1604-E1612 (2013).

64. Zhang, L. et al. TRAP1 rescues PINK1 loss-of-function phenotypes. Hum. Mol. Genet. 22, 2829-2841 (2013).

65. Alfred Witjes, J. et al. Updated 2016 EAU guidelines on muscle-invasive and metastatic bladder cancer. Eur. Urol. 71, 462-475 (2017).

66. Karim, N. F. A. et al. Phase I trial of chloroquine (CQ)/hydroxychloroquine (HCQ) in combination with carboplatin-gemcitabine (CG) in patients with advanced solid tumors. J. Clin. Oncol. 37, 3027-3027 (2019).
67. Qin, L. et al. Chloroquine enhances the efficacy of cisplatin by suppressing autophagy in human adrenocortical carcinoma treatment. Drug Des. Devel. Ther. 10, 1035-1045 (2016).

68. $\mathrm{Yu}, \mathrm{L}$. et al. Induction of autophagy counteracts the anticancer effect of cisplatin in human esophageal cancer cells with acquired drug resistance. Cancer Lett. 355, 34-45 (2014).

69. Zhang, H. Q. et al. Antitumor activity of chloroquine in combination with Cisplatin in human gastric cancer xenografts. Asian Pac. J. Cancer Prev. 16, 3907-3912 (2015).

70. Zhao, X. G. et al. Chloroquine-enhanced efficacy of cisplatin in the treatment of hypopharyngeal carcinoma in xenograft mice. PLoS ONE 10, e0126147 (2015).

71. Hoogenboezem, E. N. \& Duvall, C. L. Harnessing albumin as a carrier for cancer therapies. Adv. Drug Deliv. Rev. 130, 73-89 (2018).

72. Barrera, M., Koob, S., Dikov, D., Vogel, F. \& Reichert, A. S. OPA1 functionally interacts with MIC60 but is dispensable for crista junction formation. FEBS Lett. 590, 3309-3322 (2016).

73. Hieke, N. et al. Expression of a ULK1/2 binding-deficient ATG13 variant can partially restore autophagic activity in ATG13-deficient cells. Autophagy 11, 1471-1483 (2015).

74. Skowron, M. A. et al. Distinctive mutational spectrum and karyotype disruption in long-term cisplatin-treated urothelial carcinoma cell lines. Sci. Rep. 9, 14476 (2019).

75. Del Gaudio, F. et al. Chemoproteomic fishing identifies arzanol as a positive modulator of brain glycogen phosphorylase. Chem. Commun. 54, 12863-12866 (2018).

76. Shevchenko, A., Tomas, H., Havlis, J., Olsen, J. V. \& Mann, M. In-gel digestion for mass spectrometric characterization of proteins and proteomes. Nat. Protoc. 1, 2856-2860 (2006). 